\title{
Hypomorphism of Fto and Rpgrip1/ causes obesity in mice
}

\author{
George Stratigopoulos, ${ }^{1}$ Lisa Cole Burnett, ${ }^{1}$ Richard Rausch, ${ }^{1}$ Richard Gill, ${ }^{1}$ David Barth Penn, ${ }^{2}$ Alicja A. Skowronski, \\ Charles A. LeDuc, ${ }^{1}$ Anthony J. Lanzano, ${ }^{3}$ Pumin Zhang, ${ }^{4}$ Daniel R. Storm, ${ }^{5}$ Dieter Egli, ${ }^{1}$ and Rudolph L. Leibel ${ }^{1}$ \\ 'Naomi Berrie Diabetes Center and Division of Molecular Cenetics, Department of Pediatrics, College of Physicians and Surgeons of Columbia University, New York, New York, USA \\ ${ }^{2}$ Alaska Family Medicine Residency, Anchorage, Alaska, USA. ${ }^{3}$ John F. Kennedy High School, Bellmore, New York, USA. ${ }^{4}$ Department of Molecular Physiology and Biophysics, \\ Baylor College of Medicine, Houston, Texas, USA. 5Department of Pharmacology, University of Washington, Seattle, Washington, USA.
}

\begin{abstract}
Noncoding polymorphisms in the fat mass and obesity-associated (FTO) gene represent common alleles that are strongly associated with effects on food intake and adiposity in humans. Previous studies have suggested that the obesity-risk allele rs8050136 in the first intron of FTO alters a regulatory element recognized by the transcription factor CUX1, thereby leading to decreased expression of $F T O$ and retinitis pigmentosa CTPase regulator-interacting protein-1 like (RPCRIP1L). Here, we evaluated the effects of rs 8050136 and another potential CUX1 element in rs1421085 on expression of nearby genes in human induced pluripotent stem cell-derived (iPSC-derived) neurons. There were allele-dosage effects on FTO, RPCRIP1L, and AKT-interacting protein (AKTIP) expression, but expression of other vicinal genes, including IRX3, IRX5, and RBL2, which have been implicated in mediating functional effects, was not altered. In vivo manipulation of CUX1, Fto, and/or Rpgrip1I expression in mice affected adiposity in a manner that was consistent with CUX1 influence on adiposity via remote effects on Fto and Rpgrip1/ expression. In support of a mechanism, mice hypomorphic for Rpgrip1/ exhibited hyperphagic obesity, as the result of diminished leptin sensitivity in Leprb-expressing neurons. Together, the results of this study indicate that the effects of FTO-associated SNPs on energy homeostasis are due in part to the effects of these genetic variations on hypothalamic FTO, RPGRIP1L, and possibly other genes.
\end{abstract}

\section{Introduction}

The strongest GWAS signal yet detected for common obesity is generated by SNPs located within an approximately $47-\mathrm{kb}$ interval in the first intron of fat mass and obesity-associated (FTO) that account for a 2 to 3.5 pound per allele increase in body weight in adults and children (1). Previous studies have produced conflicting results regarding the correlation of allelic variations at the implicated FTO intronic region with FTO expression or expression of nearby genes. Berulava et al. (2) reported changes in FTO expression in an intronic allele (rs9939609) dose-dependent manner in lymphocytes. In contrast, Jowett et al. (3) found an allele-dependent (rs8050136) change in expression of nearby gene retinoblastoma-like $2(R B L 2)$ but not in FTO in lymphocytes. Smemo et al. (4) reported higher Iroquois homeobox 3 (IRX3) expression in cerebellar and adipose tissue homozygous for the FTO obesity-risk allele (rs9930506) compared with tissue from individuals homozygous for the protective FTO alleles. Claussnitzer et al. (5) reported effects on allelic variant rs1421085 on Irx3 and Irx5 expression in preadipocytes, but no allelicspecific effects in expression were seen in a hippocampal neuronal cell line. The inconsistencies in correlation of allelic variation to expression of FTO and vicinal genes may be attributed to the possibility that different haplotypes affect different genes. This would be consistent with multiple mechanisms underlying

Conflict of interest: The authors have declared that no conflict of interest exists. Submitted: November 16, 2015; Accepted: February 25, 2016.

Reference information: J Clin Invest. 2016;126(5):1897-1910. doi:10.1172/JCI85526. such a highly penetrant effect on adiposity. It may also reflect limited physiological relevance and/or developmental stage of the cells as well as genetic heterogeneity among subjects.

FTO shares protein motifs with $\mathrm{Fe}$ (II) and 2-oxoglutaratedependent demethylases of single-stranded RNA and DNA (6). FTO RNA demethylase activity has been linked with regulating dopamine signaling (7), ghrelin production (8), and adipogenesis (9). Nevertheless, nonsynonymous variants in FTO are found equally in obese and lean individuals, suggesting that FTO protein itself may not be the sole cause for the association of the FTO locus with obesity in humans (10-12). Claussnitzer et al. (5) proposed a mechanism in which obesity-risk alleles of rs1421085 in human mesenchymal adipocyte progenitors result in decreased "beiging" (thermogenic potential) of new adipocytes. Nevertheless, the relevance of this effect in humans is unclear, as the effects of the obesity-risk alleles in the first intron of FTO have been consistently reported to convey effects on food intake and not reduced energy expenditure (for example, refs. 13-15).

Retinitis pigmentosa GTPase regulator-interacting protein-1 like ( $R P G R I P 1 L)$, located $<100$ bp upstream and in opposite transcriptional orientation to FTO (Figure 1A), is a transition zone structural component of the primary cilium (16), an organelle implicated in human energy homeostasis (17). We have shown that the obesity-protective allele at FTO intronic SNP rs8050136 promotes the DNA-binding of the cut-like homeobox 1 (CUX1) isoform P110 that acts as an activator of FTO and RPGRIP1L expression in vitro $(18,19)$. In contrast, the obesity-risk allele at rs8050136 is preferentially occupied by CUX1 isoform P200, 
which acts as a transcriptional repressor of FTO in vitro (19). We have also reported that Fto and Rpgripll expression is downregulated in the arcuate hypothalami of fasted wild-type (C57BL/6J) mice (19) and that activity of hypothalamic cathepsin L, the protease that cleaves P200 to generate P110 (20), is decreased by fasting (19). A prediction from these findings is that the relative increase in P200 and decrease in P110 resulting from low cathep$\sin \mathrm{L}$ activity leads to reduced RPGRIP1L/FTO expression and increased food intake. RPGRIP1L/FTO expression is decreased in leptin-deficient $\left(L e p^{\mathrm{ob}}\right)$ mice and upregulated by leptin administration in these animals, indicating that RPGRIP1L/FTO may have anorectic effects via the canonical leptin signaling pathway (18, 19). Mice systemically deleted for one Rpgrip1l allele (Rpgrip1 $1 l^{+/}$ mice; ref. 21) and Fto-null mice (22) display normal circulating leptin levels. Nevertheless, leptin receptor (LEPR) molecules localize in the trans-Golgi network next to the cilium in hypothalamic neurons of mice administered leptin; this "convening" of LEPR is reduced by fasting in vivo, by Rpgripll knockdown in a neuronal cell line, and in the arcuate hypothalamus of Rpgrip1 $1^{+/}$ mice $(19,21)$. Based on these findings, we hypothesized that the FTO obesity-risk allele at rs8050136 alters CUX1 binding, leading to decreased hypothalamic RPGRIP1L/FTO, diminished leptin sensitivity, and increased food intake.

In the present study, we examined the effects of the obesity-risk alleles at rs8050136/rs1421085 (CUX1-binding sites) in the first intron of FTO on CUX1 binding and RPGRIP1L and FTO expression in human iPSC-derived neurons; the role of CUX1 in regulating the expression of RPGRIP1L, FTO, and nearby genes in vivo; the effects of RPGRIP1L/FTO hypomorphism on leptin sensitivity; and the tissue-specific effects of RPGRIP1L hypomorphism in energy homeostasis.

\section{Results}

Alleles of rs1421085 in FTO alter CUX1 binding. Cohorts of individuals of African descent have been examined by others in an effort to reduce the size of the FTO interval implicated in adiposity (2325). In these studies, rs 8050136 (minor allele frequency $=0.34$ ), and the landmark SNP rs9939609 first reported by McCarthy (1), were associated weakly or not at all with BMI in subjects of African descent. However, haplotypes with SNPs rs3751812 ( 2 kb $3^{\prime}$ and $5^{\prime}$ of rs8050136 and rs9939609, respectively), rs9941349 ( $9 \mathrm{~kb} 3^{\prime}$ of rs8050136), and rs1421085 ( 15 kb 5' of rs8050136; minor allele frequency $=0.38$ in Europeans and 0.09 in African Americans, respectively) were associated with BMI in African Americans (Figure 1A). Like rs8050136, rs1421085 is embedded within a binding site for the transcription factor CUX1 (23). Based on our previous work, the rs8050136 A (obesity-risk) allele preferentially binds the P200 CUX1 isoform, whereas the rs8050136 C (protective) allele preferentially binds the P110 isoform (19). By gel-shift assay, P200 bound the rs1421085 site weakly, presumably due to lack of conservation of the CR1 domain core binding sequence (ATA) that dictates specificity of P200 (Figure 1B and Supplemental Figure 1A; supplemental material available online with this article; doi:10.1172/JCI85526DS1). P200 preferentially bound the $\mathrm{C}$ (obesity-susceptibility) allele at rs1421085, because $\mathrm{C}$ is the preferred nucleotide at the CR1-binding motif (ref. 26 and Supplemental Figure 1A). P110 had higher affinity for the rs1421085 site than $\mathrm{P} 200$, because the core recognition ATC sequence for the HD domain, which dictates specificity of P110 (19), is conserved (Supplemental Figure 1A). P110 preferentially bound the protective $(\mathrm{T})$ allele at rs1421085, presumably because it conforms to the binding motif for CR2 and CR3 (ref. 26, Figure 1B, and Supplemental Figure 1A). We further assessed the effects of these allelic variants on the putative RPGRIP1L and FTO minimal promoters (Figure 1C). Promoter probing by luciferase assay revealed that P200 decreases the activity of the FTO putative minimal promoter (19) in the presence of the rs1421085 C (obesity-risk) allele, whereas P110 increases the transcriptional activity of the RPGRIP1L and FTO minimal promoters in the presence of the genomic site that includes the rs1421085 T (protective) allele compared with the C (obesitysusceptibility) allele. The diminished binding of the P200 transcriptional repressor and the increased affinity of the P110 transcriptional activator for the rs1421085 obesity-risk and -protective alleles, respectively, are consistent with RPGRIP1L/FTO underexpression driving the association of the FTO intronic locus with increased body weight.

rs1421085/rs8050136 correlate with RPGRIP1L/FTO/AKTIP expression. To further test our hypothesis regarding the impact on RPGRIP1L/FTO expression per obesity-risk allele at rs1421085/ rs8050136, we cultured primary fibroblasts from individuals heterozygous or homozygous for the obesity-risk or -protective alleles at rs1421085/rs8050136 (rs1421085 is in complete linkage disequilibrium with rs8050136 in these subjects). Given the proposed role of RPGRIP1L in CNS-mediated body weight regulation (21), we created iPSCs from these fibroblasts and confirmed their pluripotency (Supplemental Figure 1, B and C). We differentiated the iPSCs into neurons that we subsequently sorted for the neuronal marker CD56 (Supplemental Figure 1D) to generate cultures enriched for neuronal cells (Supplemental Figure 1E). In these neurons, FTO, RPGRIP1L, and AKT-interacting protein (AKTIP) expression was decreased per obesity-risk allele by approximately $30 \%, 25 \%$, and $15 \%$, respectively (Figure 1D). In contrast, expression of other nearby genes, IRX3, RBL2, IRX5, and chromodomain helicase DNA-binding protein 9 (CHD9), did not correlate with genotype (Supplemental Figure $1 \mathrm{~F}$ ). These findings are in agreement with the proposed effects of the obesity-risk alleles at rs1421085/rs8050136 on the regulation of RPGRIP1L/FTO by CUX1 isoforms, P200/P110.

CUX1 P200 regulates FTO expression and body composition. We further explored the predicted role of CUX1 isoform P200 as a transcriptional repressor of FTO in vivo. Five-week-old male mice congenitally overexpressing human $P 200$ knocked into the hypoxanthine-guanine phosphoribosyltransferase (Hprt) locus (27) (Hprt-P2OO mice; Supplemental Figure 2A) were approximately $10 \%$ heavier and had an approximately $100 \%$ increase in fat mass (70\% increase in percentage body fat; Figure 2A and Supplemental Figure 2B). Energy expenditure in Hprt-P2OO mice was decreased by approximately $15 \%$ and calorie intake was increased by approximately $20 \%$ compared with that in $+/+$ mice (Figure $2 \mathrm{~A})$. This was accompanied by a $25 \%$ decrease in hypothalamic Fto expression, whereas expression of Rpgrip1l and vicinal genes was not statistically different from that of $+/+$ controls (Figure 2B and Supplemental Figure 2C). This finding is consistent with our earlier in vitro studies implicating P200 as a transcriptional 
A

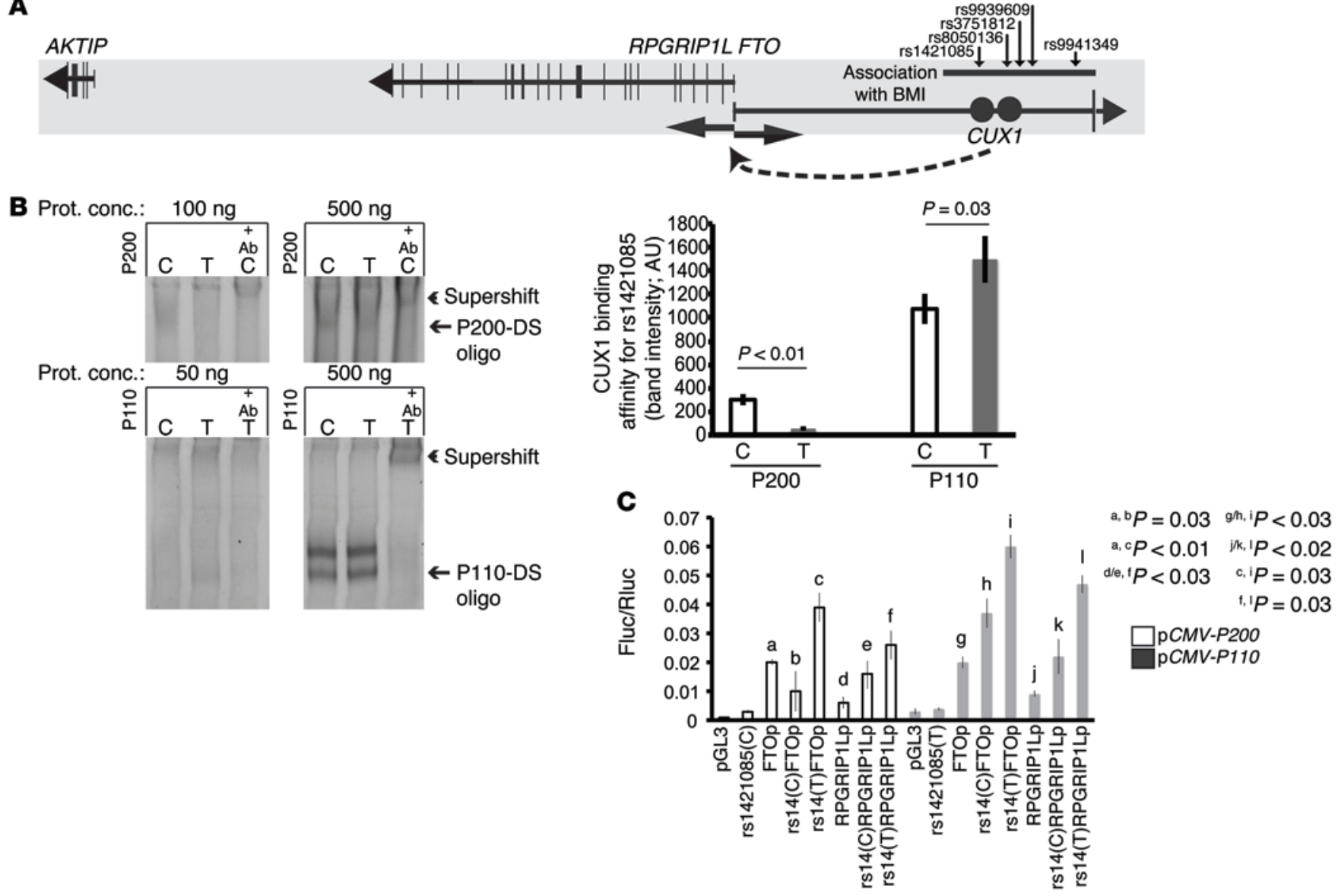

\section{D rs1421085- (C) obesity-risk, (T) protective rs8050136- (A) obesity-risk, (C) protective}
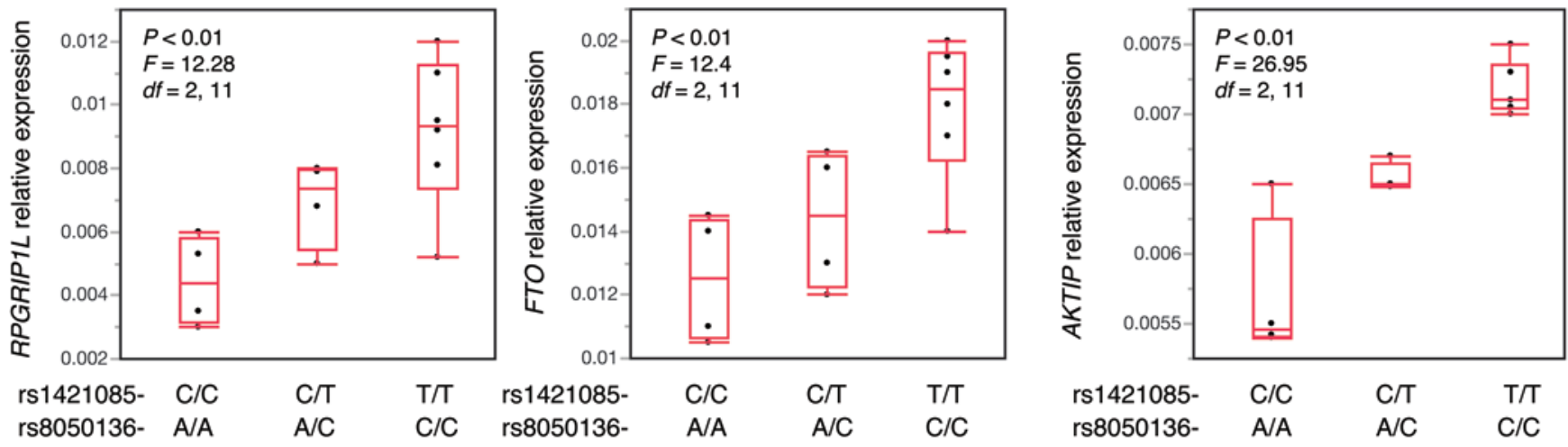

Figure 1. Functional consequences of rs1421085/rs8050136 on expression. (A) RPGRIP1L/FTO/AKTIP genomic location and implicated SNPs. (B) Nonradioactive gel-shift assay with double-stranded (DS) oligonucleotides carrying the obesity-risk C or -protective T allele at rs1421085 mixed with N2a cellular extracts overexpressing P200 or P110. P200 has higher affinity for the rs1421085 C allele, whereas P110 has higher affinity for the rs1421085 T allele. Each error bar represents 3 biological replicates. The experiment was repeated twice. (C) A luciferase assay was used to measure promoter activity. $P 200$ overexpression (CMV-P2O0) repressed FTO minimal promoter (FTOp) activity in the presence of the putative enhancer sequence carrying the C obesity-risk allele [rs14(C)FTOp], whereas P200 overexpression failed to repress the FTO promoter in the presence of the putative enhancer carrying the T protective allele [rs14(T)FTO]. FTO promoter activity was enhanced upon P110 overexpression (CMV-P110) in the presence of the enhancer carrying the rs1421085 C allele and even more so in the presence of the enhancer carrying the rs1421085 T allele, to which P110 binds with higher affinity than the $C$ allele in vitro. $P 110$ overexpression also increased activity of the RPGRIP1L minimal promoter (RPGRIP1Lp) in the presence of the putative enhancer containing the rs1421085 T allele [rs14(T)RPGRIP1Lp] more so than in the presence of the putative enhancer carrying the rs1421085 C allele [rs14(C)RPGRIP1Lp]. P200 overexpression failed to repress RPGRIP1L promoter activity in the presence of the enhancer carrying the $C$ or $T$ allele. Error bars represent 4 biological replicates. (D) Allele dose-dependent expression of FTO, RPGRIP1L, and AKTIP in neurons heterozygous $(n=4)$ or homozygous for the obesity-risk $(C / A)(n=4)$ or -protective $(T / C)(n=6)$ alleles at rs1421085 and rs8050136. In box-and-whisker plots, horizontal bars indicate the medians, boxes indicate 25 th to 75 th percentiles, and whiskers indicate 10th and 90th percentiles. Error bars represent SEM. Statistical significance was determined by 2-tailed paired Student's $t$ test or ANOVA. $P$ a values lower than 0.05 were considered significant. 

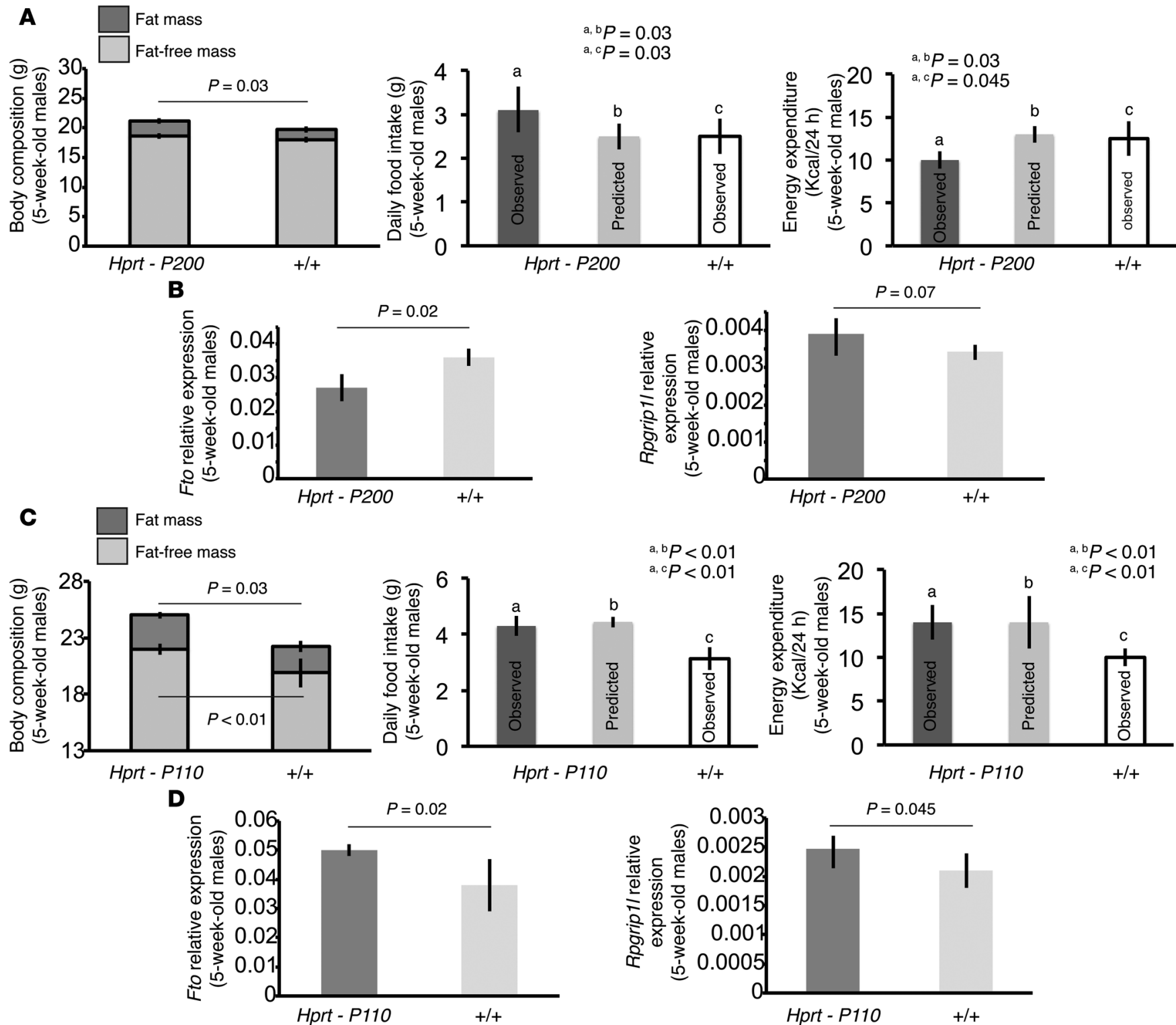

Figure 2. Systemic overexpression of CUX1 P200 and P110. (A) Increased fat mass, increased observed food intake, and lower observed energy expenditure of $P 200$-overexpressing ( Hprt-P200) ( $n=7)$ mice compared with that in control $(n=8)$ mice. The predicted energy expenditure of $H p r t-P 200$ mice, relating to fat and lean mass, was similar to that of control mice. Error bars represent SEM. Predicted values were calculated by baseline regression (see Statistics in Methods). (B) Decreased hypothalamic Fto expression in Hprt-P2OO $(n=5)$ and control $(n=5)$ mice. (C) Increased fat mass, lean mass, and observed food intake in P110-overexpressing (Hprt-P110) $(n=9)$ mice compared with control $(n=10)$ mice. The increase in observed energy intake and energy expenditure of Hprt-P110 mice was identical to the predicted energy intake relating to the increase in fat and lean mass of $H p r t-P 200$ mice, suggesting that Hprt-P110 mice are not hyperphagic or hypermetabolic. (D) Increased hypothalamic Rpgrip1l and Fto expression in Hprt-P110 ( $n=5$ ) mice compared with that in control $(n=5)$ mice. Error bars represent SEM. Statistical significance was determined by a 2-tailed paired Student's $t$ test, $P<0.05$. See also Supplemental Figure 2.

repressor of FTO but not of RPGRIP1L (19) and suggests that P200 decreases energy expenditure and increases food intake by downregulating hypothalamic expression of Fto.

The Hprt-P200 mouse is a good genetic model for studying the role of FTO on energy homeostasis, as the small change in hypothalamic Fto expression recapitulates the effect of the obesity-risk alleles on FTO expression in human neurons. Nevertheless, overexpressing P200 in vivo was not expected to fully recapitulate the molecular consequences of segregating for the obesity-risk alleles at rs8050136/rs1421085, because hypothalamic CUX1 isoform P110 protein levels were also increased in Hprt-P200 mice (Supplemental Figure 2A), presumably as a result of processing of P200 substrate by cathepsin $\mathrm{L}(18,19)$. In contrast, fasting decreases cathepsin $\mathrm{L}$ activity (19), leading to decreased Fto and Rpgripll expression due to increased P200 (repressor of FTO) and decreased P110 protein levels (activator of RPGRIP1L/FTO). Similarly, individuals segregating for obesity-risk alleles at rs8050136/rs1421085 are predicted to have higher P200 DNA-binding affinity and lower P110 DNA-binding affinity, resulting in decreased RPGRIPIL/FTO expression, as observed in iPSC-derived human neurons in vitro (Figure 1D). 

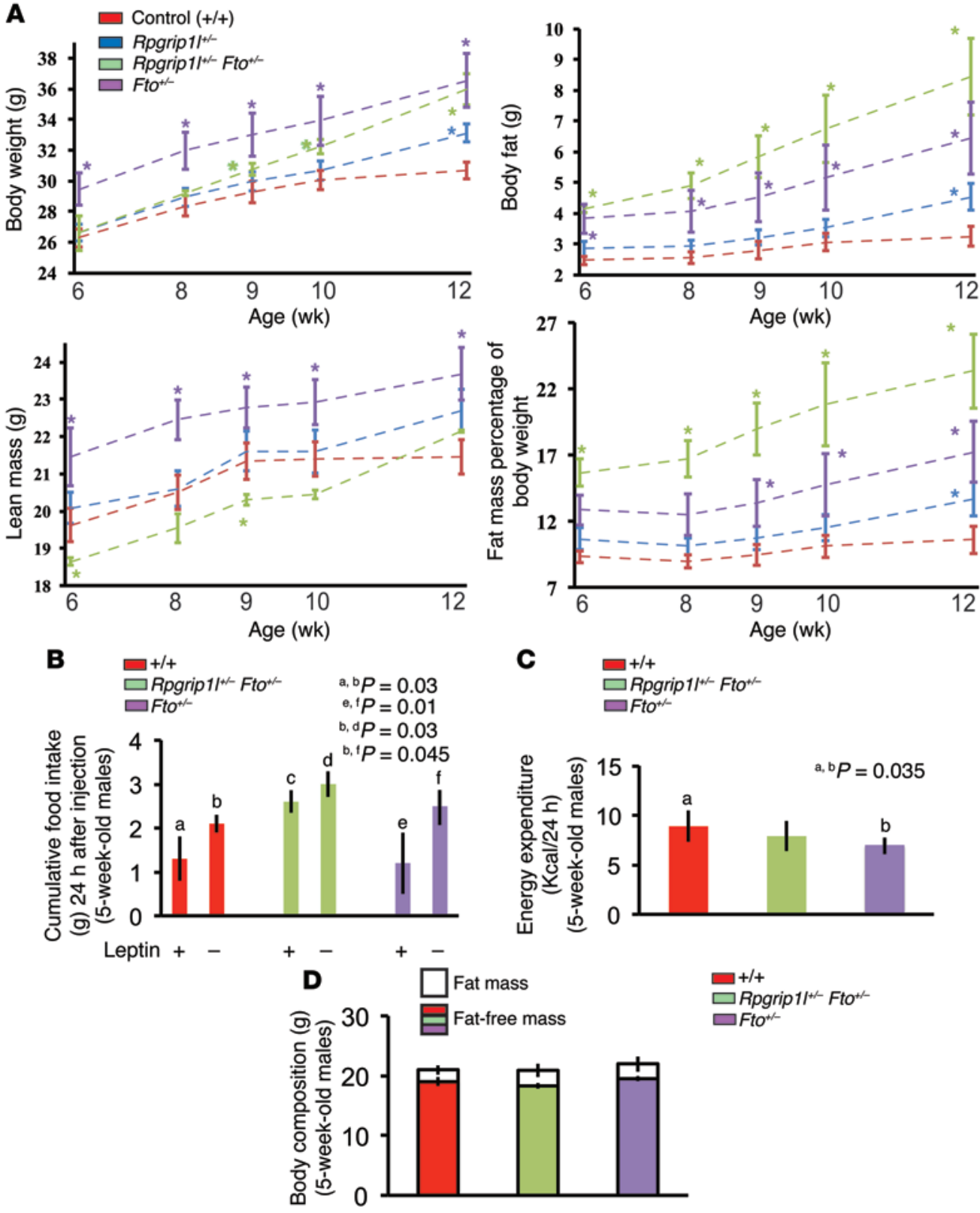

Figure 3. Phenotypic analysis of mice hypomorphic for Rpgrip1I and/or Fto. (A) Time course showing increased body weight, total fat mass, lean mass, and fat mass adjusted for body weight in systemic Rpgrip1/ heterozygous (Rpgrip $\left.11^{+-}\right)(n=8)$, Fto heterozygous $\left(\mathrm{Fto}^{+/-}\right)(n=9)$, and Rpgrip1l Fto compound heterozygous $\left(\right.$ Rpgrip11+/- $\left.\mathrm{Fto}^{+/-}\right)(n=8)$ mice compared with wild-type $(+/+)$ $(n=12)$ mice. (B) 24-hour food intake of $\mathrm{Rpgrip}^{1+/-} \mathrm{Fto}^{+/-}$and control mice administered leptin or saline ( $n=8$, respectively) as well as $\mathrm{Fto}^{+/-}$and control mice administered leptin or saline ( $n=8$, respectively). (C) Comparable energy expenditure and (D) body composition of 5-week-old Rpgrip11+/- $\mathrm{FtO}^{+/-}(n=8), \mathrm{Fto}^{+/-}$ $(n=8)$, and $+/+(n=8)$ mice. Error bars represent SEM. Statistical significance was determined by a 2-tailed paired Student's $t$ test, ${ }^{*} P<0.05$. See also Supplemental Figure 3.
CUX1 P110 regulates RPGRIP1L/FTO expression and body growth. Systemic overexpression of P110 in mice (Hprt-P110 mice; ref. 28 and Supplemental Figure 2A) resulted in approximately $30 \%$ and approximately $13 \%$ increases in hypothalamic Fto and Rpgrip1l expression, respectively, but did not affect the expression of other vicinal genes (Figure 2D and Supplemental Figure 2D). Surprisingly, the changes in hypothalamic Fto and Rpgrip1l expression were accompanied by approximately $10 \%$ and $30 \%$ proportional increases in fat-free mass and fat mass, respectively (Figure 2C). The percentage of body fat was, however, indistinguishable between Hprt-P11O and control mice (Supplemental Figure 2B), suggesting that the increase in Rpgrip1l/Fto expression in HPRT-P110 mice results in increased somatic growth (lean and fat) rather than elevated adiposity per se. In agreement with this formulation, the increase in lean mass accounted for the approximately $20 \%$ and $40 \%$ increases in food intake and energy expenditure, respectively (Figure $2 \mathrm{C}$ ). In the aggregate, these data support a role for P110 as an activator of RPGRIP1L/FTO expression and suggest that chronic, increased RPGRIP1L/FTO hypothalamic expression correlates with increased food intake and growth of lean and fat mass but not increased fractional fat mass (adiposity) per se.

Fto hypomorphism affects body composition. The reduction of hypothalamic FTO in fasted mice $(18,19)$, and the in vivo findings presented above indicating that $\mathrm{P} 2 \mathrm{OO}$ overproduction decreased hypothalamic Fto expression and was associated with decreased energy expenditure and increased food intake, prompted us to test the hypothesis that Fto hypomorphism per se increases adiposity. We measured the body composition of mice systemically deleted for exon 3 of one Fto wild-type allele $\left(\mathrm{Fto}^{+-}\right)$(29), as loss of both Fto copies results in severe loss of lean mass in mice and humans (2931). $\mathrm{Fto}^{+/}$male mice fed standard chow until the age of 14 weeks were approximately $16 \%$ heavier than control $(+/+)$ mice (Figure $3 \mathrm{~A} ; \mathrm{P}<0.05)$. Strikingly, $\mathrm{Fto}^{+/-}$mice had $71 \%$ more total fat than $+/+$ mice $(P<0.05)$ and $47 \%$ more fractional fat mass than $+/+$ controls, 


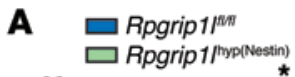

Rpgrip $1^{\text {/nW }}$
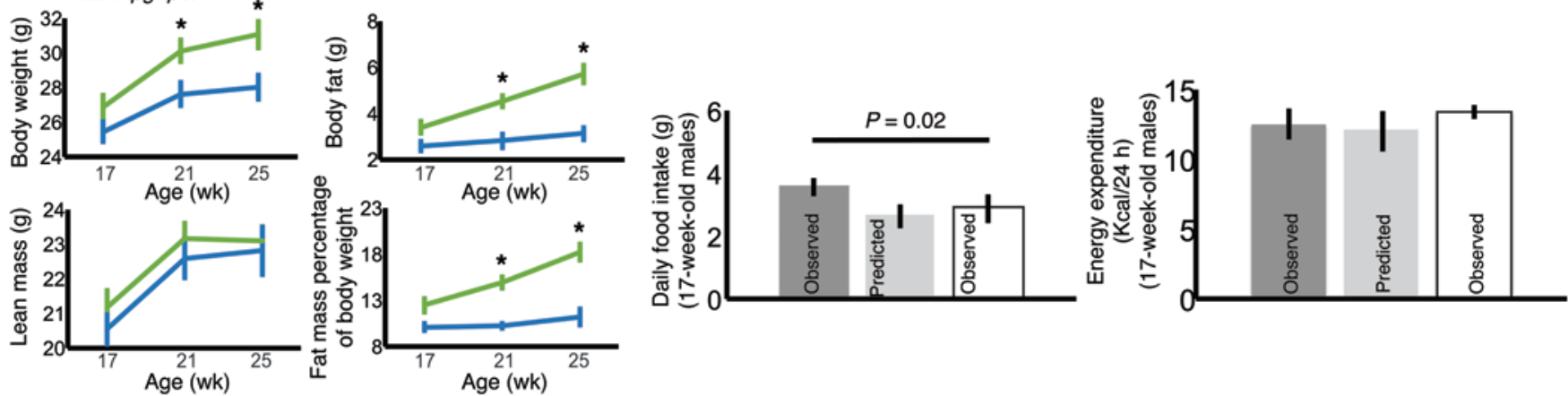

B

Fat mass $\square$ Fat-free mass

$P<0.01$
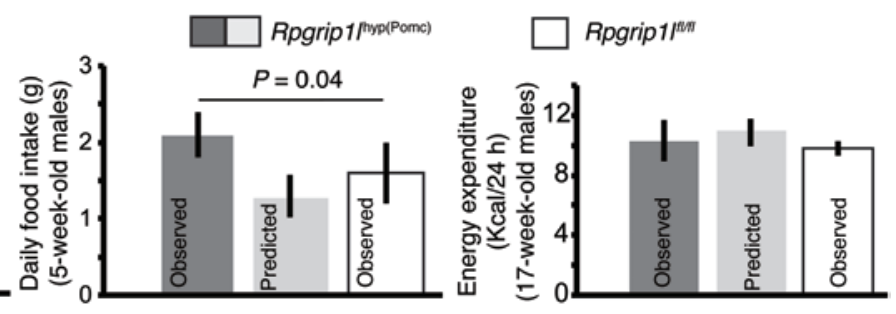

Rpgrip1/myp(Pomc) Rpgrip1/m
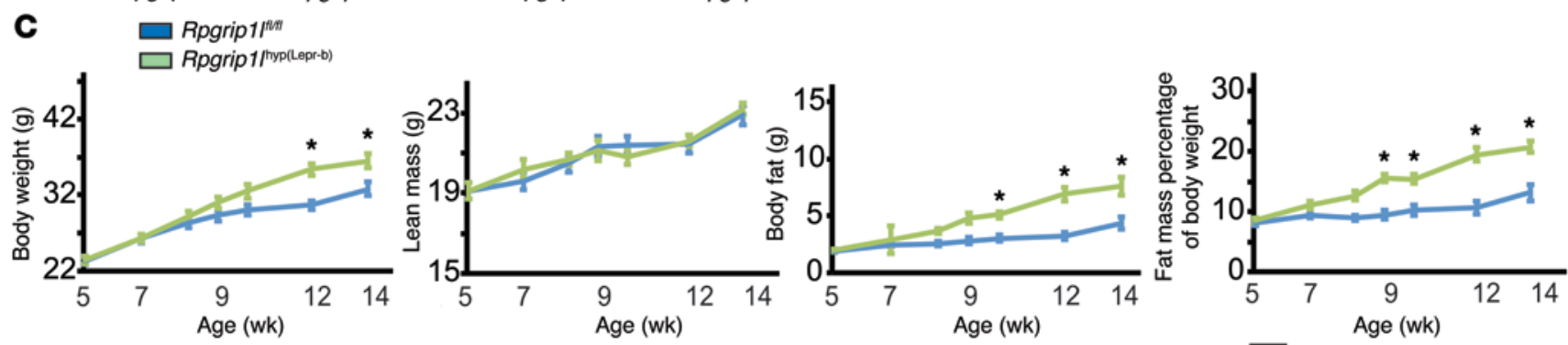

D
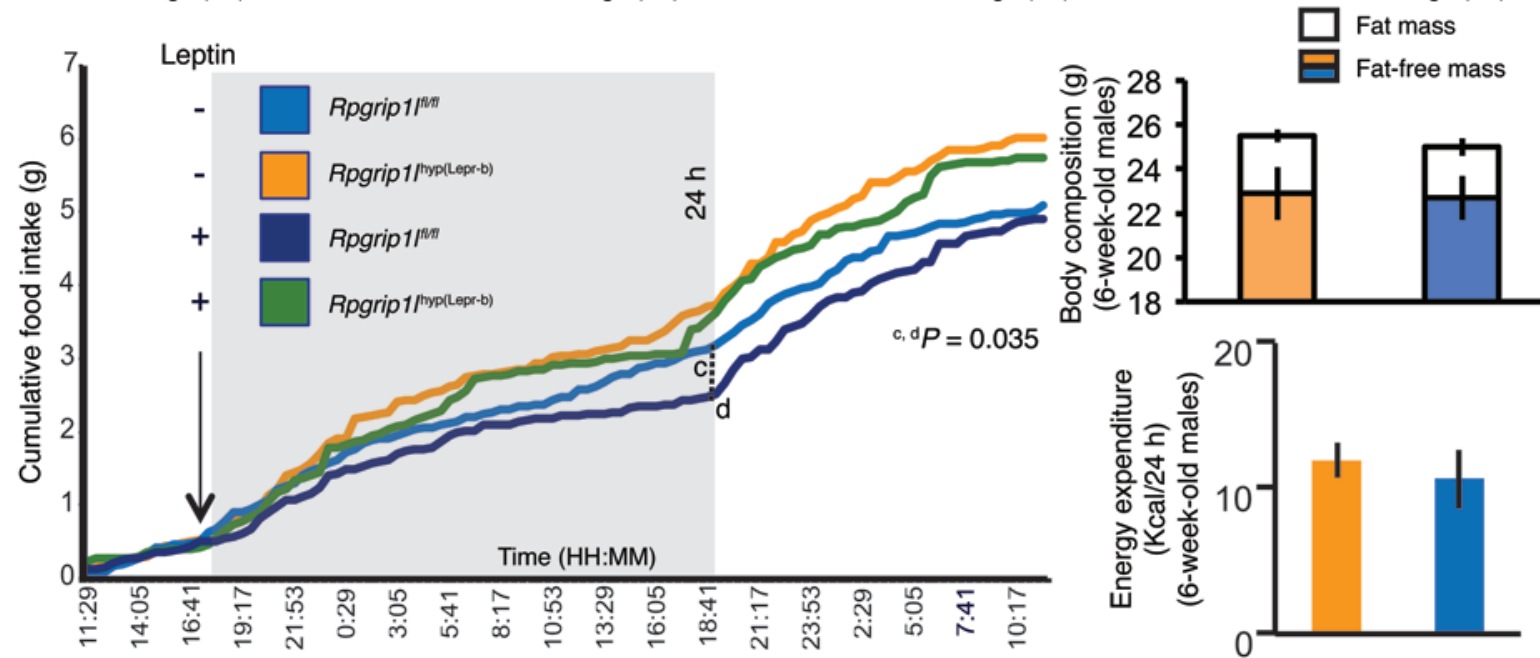

Figure 4. Phenotypic analysis of Rpgrip1/ hypomorphic mice in Pomc/Lepr-b neurons. (A) Increased body weight, total fat mass, fat mass adjusted for body weight, and food intake (observed but not predicted) of Rpgrip1l hypomorphic mice in Nestin-expressing neurons [Rpgrip $1{ }^{\text {hyp(Nestin)] }}(n=7)$ compared with those in control mice $(n=5)$ fed a LFD ad libitum. No changes were recorded in energy expenditure. (B) Increased fat mass in 5-week-old Rpgrip1I hypomorphic mice in Pomc-expressing neurons [Rpgrip1/hyp(Pomc)] $(n=8)$ compared with Rpgrip $1 f^{f / f f}$ control mice $(n=10)$. 16-week-old Rpgrip 1 hyp(Pomc) mice had increased fat and lean mass. Rpgrip $1 /$ hyp(Pomc) mice $(n=7)$ consumed more calories as early as 5 weeks of age compared with $R p g r i p 1 f^{f / f f}$ controls $(n=9)$.

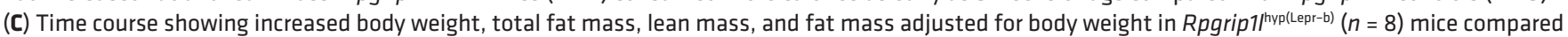
with control Rpgrip $71^{f / f \mid f l}$ mice $(n=8)$. (D) Time course of cumulative food intake in Rpgrip $71^{\text {hyp(Lepr-b) }}$ and Rpgrip $1 f^{f / f f}$ mice administered leptin $(n=8$, respectively) or saline ( $n=8$, respectively). Rpgrip $1 /^{\text {hyp(Lepr-b) }}$ mice displayed diminished leptin sensitivity compared with Rpgrip $11^{f / / f f}$ mice. Body composition and energy expenditure of 6-week-old male mice was comparable that prior to leptin or saline treatment. Error bars represent SEM. Statistical significance was determined by a 2-tailed paired Student's $t$ test, ${ }^{*} P<0.05$. See also Supplemental Figure 4. 

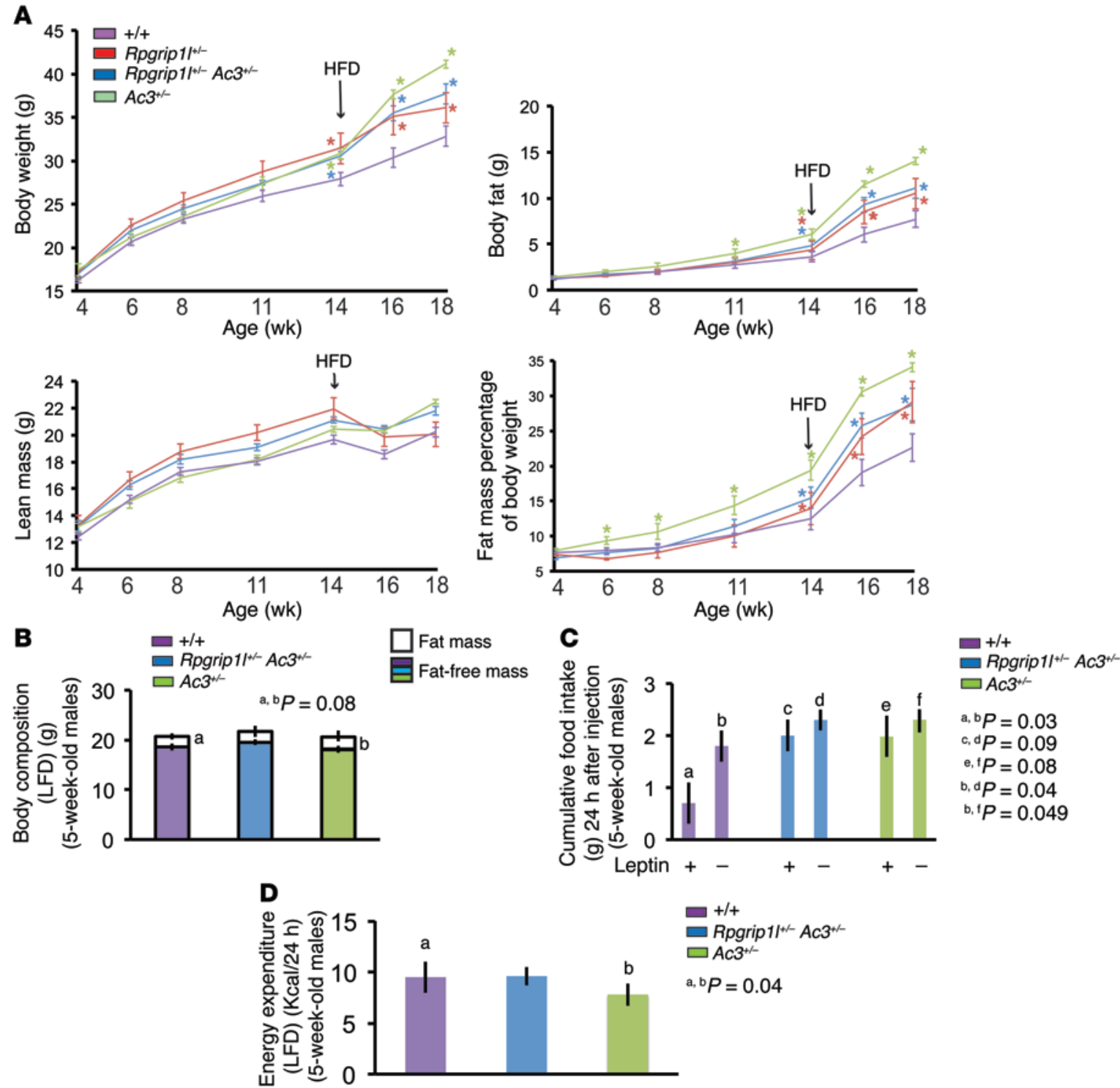

$$
\begin{aligned}
\text { a, b } P & =0.03 \\
\text { c, } d P & =0.09 \\
\text { e, } P & =0.08 \\
\text { b, d } P & =0.04 \\
\text { b, } P & =0.049
\end{aligned}
$$

Figure 5. Phenotypic analysis of mice hypomorphic for Rpgrip1l and/or Ac3. (A) Time course of body weight, total fat mass, lean mass, and fat mass adjusted for body weight in systemic Rpgrip1l heterozygous (Rpgrip $\left.11^{+/-}\right)(n=9), A c 3$ heterozygous $\left(A c 3^{+/-}\right)(n=9), R p g r i p 11$ Ac3 compound

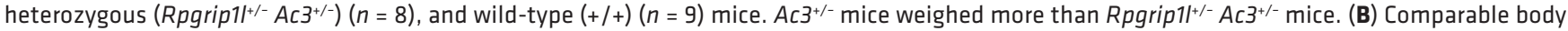

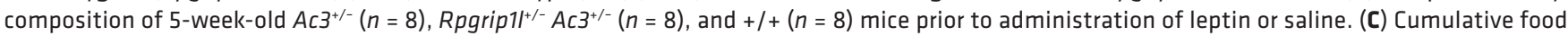
intake of 5-week-old $A c 3^{+/-}$, Rpgrip $11^{+/-} A c 3^{+--}$, and $+/+$mice treated leptin $\left(n=8\right.$, respectively) or saline $\left(n=8\right.$, respectively). (D) $A c 3^{+/-}$mice $(n=8)$ displayed lower energy expenditure than $+/+$ and $R$ pgrip $11^{+/-} A c 3^{+/-}$mice $(n=8)$. Error bars represent SEM. Statistical significance was determined by a 2-tailed paired Student's $t$ test, ${ }^{*} P<0.05$. See also Supplemental Figure 5.

respectively $(P<0.05)$. Effects on lean mass accounted for some of the differences in fractional fat mass: lean mass was increased by $9 \%$ in the $\mathrm{Fto}^{+/-}$mice $(P<0.05)$. In the aggregate, these data suggest that Fto hypomorphism increases adiposity in animals.

Rpgrip1l/Fto compound heterozygosity has additive effects on adiposity. We have previously shown that Rpgripll hypomorphism increases food intake and adiposity (21). Based on our finding that the obesity-risk alleles at rs8050136 and rs1421085 correlate with decreased RPGRIP1L/FTO expression in human neuronal cells, we examined the effects on adiposity of RPGRIPIL/FTO compound hypomorphism in vivo. Fourteen-week-old compound heterozygous Rpgrip1l/Fto male mice (Rpgrip1 ${ }^{+-} \mathrm{Fto}^{+/-}$) and Rpgrip1l heterozygous mice (Rpgrip1 $\left.l^{+-}\right)$fed standard chow had $130 \%$ and
$17 \%$ increases, respectively, in total fat mass compared with that in $+/+$ mice (Figure 3A; $P<0.05$ ). Fractional fat mass was increased $(125 \%)$ as well $(P<0.05)$. Given the $50 \%$ increase in fractional fat mass of 14-week-old $\mathrm{Fto}^{+/-}$mice (Figure 3A), this finding suggests additive effects of Rpgrip1l and Fto on adiposity.

Diminished leptin sensitivity in Rpgripll ${ }^{+-} \mathrm{Fto}^{+-}$mice. The increased food intake of Rpgrip1 $l^{+/}$mice is accompanied by diminished responses of arcuate neurons to i.p. administration of leptin (21). We examined leptin sensitivity in $\mathrm{Rpgripl}^{+/-} \mathrm{Fto}^{+/-}$and $\mathrm{Fto}^{+/-}$mice at 5 weeks of age, when no differences in body mass or composition were present (Figure 3D). As expected, the 24-hour cumulative food intake of $+/+$ mice administered i.p. leptin was decreased by $35 \%(0.7 \mathrm{~g}$; $2.6 \mathrm{Kcal}$ ) (Figure 3B; a,b $P=0.03)$. Simi- 


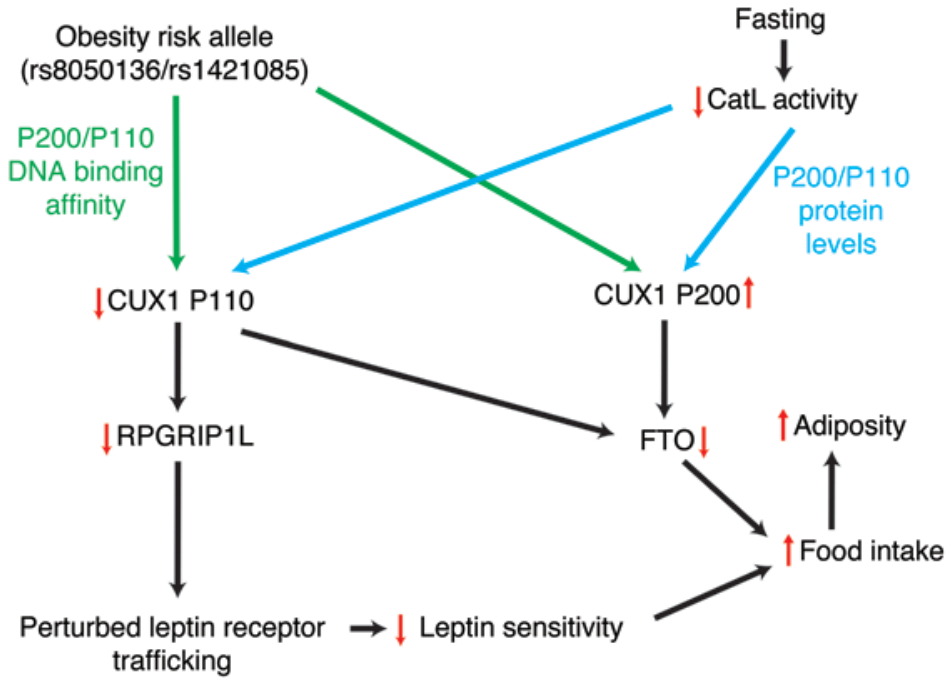

Figure 6. Molecular basis of obesity susceptibility at the FTO locus. rs 8050136 and rs1421085 risk alleles increase the binding affinity of P200 and decrease that of P110 CUX1 proteins, thus mimicking the fasting state in which lower hypothalamic cathepsin L (CatL) activity results in increased $\mathrm{P} 200$ and decreased $\mathrm{P} 110$ protein levels because of reduced proteolytic cleavage of the long P200 isoform into the shorter isoform P110 (19). Increased hypothalamic P200 (FTO repressor) and decreased P110 (activator of RPGRIP1L and FTO) results in coordinate suppression of RPGRIP1L/FTO, resulting in increased food intake. larly, $\mathrm{Fto}^{+/-}$mice treated with leptin consumed approximately $50 \%$ (1.25 g; $4.7 \mathrm{Kcal}$ ) fewer calories when fed regular chow (low-fat diet [LFD]) over the subsequent 24 hours compared with $\mathrm{Fto}^{+/-}$ mice treated with saline (Figure $3 \mathrm{~B} ;{ }^{\mathrm{e}, \mathrm{f}} \mathrm{P}=0.01$ ). Thus, Fto hypomorphism, unlike Rpgrip1l hypomorphism, does not affect acute food intake responses to leptin. In contrast, the 24-hour cumulative caloric intake of Rpgrip1 $1 \mathrm{l}^{+-} \mathrm{Fto}^{+/-}$mice administered leptin i.p.

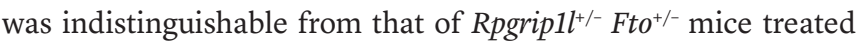
with saline (Figure 3B). These data suggest that lack of one func-

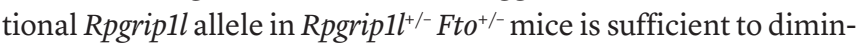
ish acute food intake suppression by leptin.

Rpgrip1l ${ }^{+/-} \mathrm{Fto}^{+/-}$and $\mathrm{Fto}^{+-}$mice and food intake and energy expenditure. Hyperphagia accounted for the increased adiposity of 5-week-old male Rpgrip1l ${ }^{+/-} \mathrm{Fto}^{+/-}$mice: specifically, these mice had a $46 \%$ greater 24 -hour cumulative caloric intake $(1 \mathrm{~g}$; $3.75 \mathrm{Kcal}$ ) (Figure $3 \mathrm{~B}$; ${ }^{\mathrm{b}, \mathrm{d}} \mathrm{P}=0.03$ ). $\mathrm{Fto}^{+/-}$mice ate $20 \%$ more calories in 24 hours $(0.4 \mathrm{~g} ; 1.5 \mathrm{Kcal})$ than $+/+$ mice at 5 weeks of age (Figure 3B; ${ }^{\mathrm{b}, \mathrm{f}} \mathrm{P}=0.045$ ). Given the $17 \%$ increase in food intake of 5-week-old Rpgrip $1 l^{+-}$mice (21), the $46 \%$ increase in caloric intake of Rpgrip1l+- $\mathrm{Fto}^{+/-}$mice appears to reflect synergistic contributions of Rpgrip1l and Fto. In addition, energy expenditure in 5-week-old male $\mathrm{Fto}^{+/-}$mice was $17 \%$ lower than that in $+/+$ mice (Figure $3 \mathrm{C}$ ). These data are in agreement with the decreased energy expenditure and increased food intake observed in Hprt-P2OO mice that display reduced hypothalamic Fto expression (Figure 2, A and B). The energy expenditures of 5-week-old Rpgrip11 ${ }^{+/-} \mathrm{Fto}^{+/-}$and +/+ mice were indistinguishable (Figure $3 \mathrm{C}$ ), suggesting that the synergistic effect of Rpgrip1l and Fto on adiposity is conveyed via increased energy intake and not decreased energy expenditure.

Hypothalamic gene expression of Rpgrip1l ${ }^{+/-} \mathrm{Fto}^{+/-}$and $\mathrm{Fto}^{+/-}$ mice. We confirmed by RT-PCR that, in Rpgrip1 ${ }^{+/-} \mathrm{Fto}^{+/-}$mice, the expression of hypothalamic Rpgrip1l and Fto - but not the other vicinal genes - was decreased by $30 \%$ and $70 \%$, respectively (Supplemental Figure $3 \mathrm{~A}$ ). In addition, we found that the

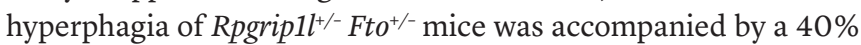
increase in neuropeptide Y (Npy) and a $40 \%$ decrease in proopiomelanocortin (Pomc) expression in the hypothalamus, in agreement with diminished leptin sensitivity in these animals
(Supplemental Figure 3B). Npy expression was increased by $30 \%$ in $\mathrm{Fto}^{+/-}$mice compared with that in $+/+$mice (Supplemental Figure 3D). Interestingly, the anticipated $50 \%$ decrease in hypothalamic Fto expression in $\mathrm{Fto}^{+-}$mice was accompanied by a $40 \%$ increase in hypothalamic Rpgrip1l expression (Supplemental Figure 3C). As loss of both copies of Fto resulted in a dramatic reduction of lean mass, whereas loss of one Fto allele increases lean mass, it is conceivable that what drives the increase of lean mass in $\mathrm{Fto}^{+/-}$mice is the upregulation of Rpgripll. This may also be the case in Hprt-P110 mice, which display upregulation of hypothalamic Rpgrip1l and increased lean mass (Figure 2, C and D). Therefore, while Rpgrip1l hypomorphism increases adiposity, Rpgrip1l overexpression may promote growth of lean mass.

Tissue-specific Rpgrip1l hypomorphism. Apparently, the role of Rpgrip1l in energy homeostasis is distinct from that of Fto, in that Rpgrip1l controls food intake by modulating LEPR physiology, whereas Fto affects energy expenditure and food intake in a leptinindependent manner. We assessed whether induced hypomorphism of Rpgripll in the developed adult brain is sufficient to drive increased energy intake and adiposity. Three i.p. injections of tamoxifen over a 5-day period in 12-week-old male mice homozygous for the Rpgrip1l floxed allele (Rpgrip1 $\left.l^{f / f l}\right)$ and segregating for an inducible Nestin-CreER ${ }^{\mathrm{T} 2}$ allele [Rpgrip1 $\left.{ }^{\text {hyp(Nestin) })}\right]$ resulted in a 70\% decrease of Rpgrip1l expression in nestin-positive hypothalamic neurons (Supplemental Figure 4A). By 21 weeks of age,

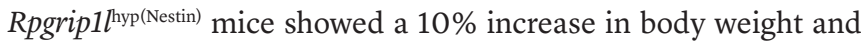
a $60 \%$ increase in fat mass associated with a $20 \% 24$-hour cumulative increase in food intake (0.6 g; $2.25 \mathrm{Kcal})$ (Figure 4A). Thus, Rpgrip1l hypomorphism in the adult CNS is sufficient to increase food intake and adiposity in mice. We also congenitally decreased

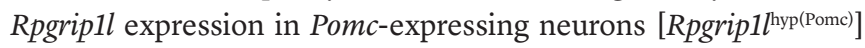
by $85 \%$ (Supplemental Figure 4B). 16-week-old Rpgrip1 $1 l^{\text {hyp(Pomc) }}$ mice fed standard chow had $100 \%$ more fat than Rpgrip $1 l^{f / f l}$ mice (Figure 4B). As early as 5 weeks of age, Rpgrip1 ${ }^{\text {hyp(Pomc) }}$ mice were hyperphagic and had $50 \%$ more fat mass, with no apparent effect on energy expenditure (Figure 4B). Congenital Rpgrip1l hypomorphism (75\% reduction in Rpgrip1l expression; Supplemental Figure $4 \mathrm{~B}$ ) in LEPR isoform b-expressing (Lepr-b-expressing) neurons of chow-fed 14-week-old mice [Rpgrip1 ${ }^{\text {hyp(Lepr-b) }}$ resulted in a $50 \%$ 
increase in fat mass (Figure 4C). Four-week-old Rpgrip1 ${ }^{\text {hyp(Lepr-b) }}$ and Rpgrip1 $l^{f / f l}$ control mice weighed the same, but energy intake of the Rpgrip $1 l^{\text {hyp (Lepr-b) }}$ mice was significantly higher (Supplemental Figure 4D). Consistent with these observations, 24-hour cumulative food intake was unaffected in 6-week-old Rpgrip1 $l^{\text {hyp(Lepr-b) }}$ mice administered i.p. leptin (Figure 4D). Conversely, 24-hour cumulative food intake was decreased by $30 \%$ (0.7 g; $2.6 \mathrm{Kcal}$; $\left.{ }^{c, d} P=0.035\right)$ in 6-week-old Rpgripl $1 l^{f / f l}$ mice (indistinguishable in body composition and energy expenditure from 6-week-old

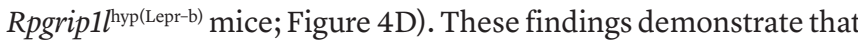
Rpgrip1l hypomorphism in Lepr-b neurons alone is sufficient to increase adiposity and diminish functional leptin sensitivity.

RPGRIP1L and AC3 in energy homeostasis. We previously reported that, in the hypothalamus of the $R$ pgrip1l ${ }^{+-}$mouse and in human fibroblasts with biallelic hypomorphic mutations of RPGRIP1L (from patients with Joubert syndrome), the number of adenylyl cyclase 3-positive (AC3-positive) cilia is diminished, accompanied by impaired leptin-stimulated convening of LEPR to the vicinity of the cilium and diminished hypothalamic pSTAT3 generation in response to leptin (21). Congenital systemic deletion of Ac3 results in obesity in mice (32). AC3 catalyzes the formation of cAMP, which in turn mediates control of aspects of energy homeostasis via the PKA/cAMP response element-binding protein (CREB) or AMP-activated protein kinase (AMPK) pathways, which are themselves leptin responsive (33, 34). We examined potential interactions of Rpgrip1l and Ac3 molecules with regard to adiposity and leptin sensitivity. We generated mice heterozygous for an $A c 3$-null allele $\left(\mathrm{Ac3}^{+/-}\right.$mice; ref. 32) and mice compound heterozygous for null alleles of Rpgrip1l and Ac3 (Rpgrip1l ${ }^{+-} \mathrm{Ac3}^{+/-}$mice) (Supplemental Figure 5, A and

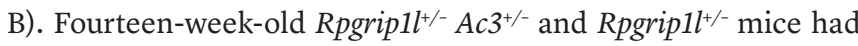
$30 \%$ higher fractional fat mass, whereas $\mathrm{Ac}^{+/-}$mice had $70 \%$ higher fractional fat mass compared with that of $+/+$ control mice (Figure 5A). Similarly, after 4 weeks of ad libitum high-fat diet

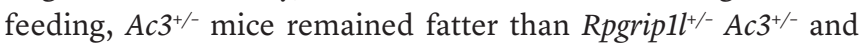
Rpgrip1 $1^{+/-}$mice. The fact that Rpgrip1l hypomorphism suppressed the adiposity in $\mathrm{Ac}^{+/-}$mice suggests that RPGRIP1L is functionally downstream of AC3 and that the AC3-mediated adiposity is not caused by RPGRIP1L hypomorphism.

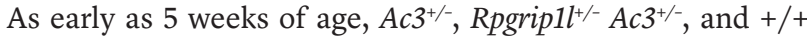
mice had similar body composition (Figure $5 \mathrm{~B}$ ), but $\mathrm{Ac}^{+/-}$and

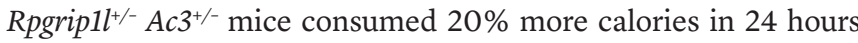
(0.4 g; $1.5 \mathrm{Kcal}$ ) than $+/+$ mice (Figure $5 \mathrm{C} ;{ }^{\mathrm{b}, \mathrm{d}} P=0.04,{ }^{\mathrm{b}, \mathrm{f}} P=0.049$ ).

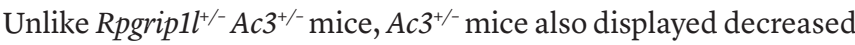
energy expenditure (Figure 5D). The observed hyperphagia in Rpgrip1 $11^{+/-} \mathrm{Ac3}^{+/-}$mice was accompanied by elevation of hypothalamic Npy transcripts, whereas $A c 3^{+/-}$mice had elevated hypothalamic Npy expression and decreased Pomc expression (Supplemental Figure 5, D and E). Both Rpgrip $1 \mathrm{l}^{+/-} \mathrm{Ac3}^{+/-}$and $\mathrm{Ac}^{+/-}$mice displayed a lack of suppression of cumulative food intake 24 hours after leptin administration (Figure 5C). Expression of genes vicinal to the Fto locus in the hypothalami of Rpgripll ${ }^{+/-} \mathrm{Ac3}^{+/-}$and $\mathrm{Ac3}^{+/-}$ mice was unaffected, with the exception of increased hypothalamic Rpgrip1l expression in $A \mathrm{C}^{+/-}$hyperphagic animals (Supplemental Figure 5, B and C), further suggesting that the Ac3-driven hyperphagia and diminished leptin sensitivity are not caused by Rpgrip1l hypomorphism.

\section{Discussion}

Prompted by previous studies implicating FTO intron 1 SNPs rs8050136 (associated with increased BMI in people of mixed European descent) and rs1421085 (associated with increased BMI in African Americans) in modulating transcription of Rpgrip1l/Fto in the CNS, we found that obesity-risk alleles of these SNPs are associated with decreased expression of FTO and the nearby gene, RPGRIP1L, in iPSC-derived human neurons. This finding is corroborated by evidence that obesity-risk alleles of rs8050136 and rs1421085 affect the binding affinity of specific isoforms (P110, P200) of the transcription factor CUX1.

We present in vivo evidence that the action of CUX1 isoform P200 results in repression of hypothalamic Fto and that the action of CUX1 isoform P110 results in activation of expression of hypothalamic Fto and Rpgrip1l (Figure 2). Expression of IRX3 and IRX5 - genes that have been implicated as the molecular mediators of the obesity-related effects of sequence variations in intron 1 of FTO - did not correlate with allelic variation at rs8050136 or rs1421085 in human iPSC-derived neurons. Expression of these genes was also unchanged in the hypothalami of mice overexpressing P200 or P110. AKTIP was the only other gene in the vicinity of the FTO obesity locus with transcript levels in iPSC-derived neurons correlated with dosage of susceptibility alleles at rs8050136 and rs1421085. In mice, hypothalamic Aktip expression trended to positive correlation with hypothalamic Rpgrip1l expression levels, consistent with possible coregulation (Supplemental Figure 2, C and D; Supplemental Figure 3C; and Supplemental Figure 5C). AKTIP is located $96.6 \mathrm{~Kb} \mathrm{3}$ ' of RPGRIP1L and is transcribed in the same orientation. RPGRIP1L, AKTIP, and FTO may form a gene cluster controlled by transcriptional elements located within the first intron of FTO. AKTIP has been implicated as a modulator of PKB (PKB or PKB/ $\mathrm{AKT}$ or $\mathrm{AKT}$ ) in the control of cell differentiation, proliferation, and apoptosis (35). It has been proposed that Rpgrip1l regulates the mitotic cell cycle in carcinogenesis (36). Given the reported effects of induced hypothalamic cell proliferation on feeding behavior (37), it is conceivable that AKTIP and RPGRIP1L may use such cell-autonomous mechanisms to regulate food intake.

Hprt-P110 mice with elevated hypothalamic Rpgrip1l expression have increased somatic growth (Figure 2C). Lean mass growth that is driven by growth hormone is regulated by hypothalamic hormones, including somatostatin (38). Somatostatin receptor 3 (SSR3) is localized to the primary cilium (39), and its function may be affected by RPGRIP1L action. It is plausible that RPGRIP1L action in hypothalamic cilia may also regulate body growth through effects on SSR3. The fact that systemic P110 overexpression also resulted in increased hypothalamic Fto expression suggests that previous reports implicating Fto in cellular nutrient sensing, adipogenesis, and ciliary function may indicate a mechanism by which FTO also affects somatic growth via the primary cilium $(9,40,41)$.

Manipulations by others of Fto gene expression in mice and rats have produced changes in body weight but not in a consistent direction: hypothalamic-specific knockdown of Fto results in increased food intake in rats (42), and energy restriction causes downregulation of Fto in the hypothalami of mice $(6,18,19,43)$. These findings are consistent with our formulation, which attributes the effects of both FTO and RPGRIP1L to suppression of food intake. In contrast, systemic Fto overexpression in mice results in 
increased fat mass (44). In the same study (44), the increase in fat mass in mice overexpressing Fto is accompanied by a significant increase in lean mass, at least in females. This is consistent with the increased somatic growth we observed in HPRT-P110 mice with elevated hypothalamic FTO expression. It has also been reported that Fto deletion protects from obesity, as Fto-null mice have reduced fat mass (22). Nevertheless, these mice also display a considerable reduction in lean mass. Moreover, late-onset systemic deletion of Fto in the adult mouse also resulted in increased total fat mass and decreased lean mass (45), and individuals lacking FTO display growth retardation $(30,31)$. In our study, congenital systemic Ftonull mice displayed reduced lean mass and elevated adiposity by 20 weeks of age (data not shown). By 24 weeks of age, there was a further reduction in lean mass and a reduction in fat mass accompanied by an increased mortality rate (data not shown). Therefore, FTO deletion may not be a good model with which to study energy homeostasis in vivo. Here, we showed that decreased hypothalamic Fto expression in $\mathrm{Hprt-P2OO}$ and $\mathrm{Fto}^{+/-}$mice resulted in increased adiposity, partly by effects on food intake, without gross effects in lean mass. These animals are more appropriate genetic models for effects of FTO hypomorphism in humans.

Similar to severe hypomorphic mutations of RPGRIP1L (46), loss of Fto in zebrafish leads to short, absent, or disorganized primary cilia and congenital abnormalities, including situs inversus, renal cystogenesis, neural crest cell defects, and microcephaly (41). Therefore, it is plausible that RPGRIP1L and FTO are coregulated as part of the same gene cluster and have synergistic effects on energy intake via ciliary morphology/function.

There are no known instances of obese patients with Joubert syndrome who lack functional RPGRIP1L. As with FTO-deficient individuals, these patients display skeletal and CNS developmental defects that may mask a role of RPGRIP1L in energy homeostasis. A recent report described an obese patient with Joubert syndrome with mutations at $A R L 13 B$ and similar brain abnormalities as those of individuals with RPGRIP1L-inactivating mutations but with no apparent skeletal anomalies (47), suggesting that CNS abnormalities caused by RPGRIP1L haploinsufficiency may cause obesity in humans.

Rpgrip1l hypomorphism in Pomc-expressing neurons increases adiposity, and Rpgrip1l hypomorphism specifically in Lepr-bexpressing neurons recapitulates the phenotype of the systemic Rpgrip1l-hypomorphic mouse, i.e., hyperphagia and increased adiposity, in association with diminished hypothalamic leptin sensitivity (21). Transient reduction of Rpgrip1l expression in the adult CNS was sufficient to increase caloric intake and adiposity, suggesting that putative developmental defects induced by congenital Rpgrip1l hypomorphism are not necessary for the Rpgrip1l effects on these phenotypes.

Previously, we found decreased numbers of Ac3-positive primary cilia in the arcuate hypothalami of systemic Rpgrip1l hypomorphic mice and in human fibroblasts homozygous for inactivating RPGRIP1L mutations (21). However, the increased food intake and diminished leptin sensitivity in Rpgrip1l hypomorphic mice does not appear to be mediated by $A c 3$, because suppression of the

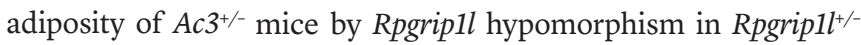
$\mathrm{Ac}^{+/-}$mice suggests that RPGRIP1L is functionally downstream of Ac3. Moreover, the fact that hypothalamic Ac3 expression was unaffected in mice in which Rpgrip1l, Fto, P200, or P110 expression was manipulated (Supplemental Figure 5A) suggests that Ac3 is not a mediator of any of the adiposity-related effects of these genes.

Claussnitzer et al. (5) showed that preadipocytes homozygous for the obesity-risk allele at $\mathrm{rs} 1421085$ present in the first intron of FTO exhibited increased IRX3 and IRX 5 expression, as a result of derepression of an enhancer site regulating these two genes. In the same study, allele-dependent enhancer activity was not observed in a hippocampal neuronal cell line (HT22) homozygous for the rs1421085 obesity-risk allele. In contrast, Smemo et al. (4) reported that homozygosity of the risk allele at rs9930506, in linkage disequilibrium with 11 BMI-associated FTO SNPs, including rs1421085 and rs8050136, was associated with elevated IRX3 expression in cerebellar tissue from European adults. In the same study, among SNPs that had brain- or adipose-specific IRX3 expression quantitative trait loci (eQTLs) in the GIANT Consortium data, the brain-specific IRX3 eQTLs showed the strongest association with BMI. These data suggest that IRX3 may affect adiposity via the CNS. In contrast, here we report that IRX3/ IRX5 expression is not associated with rs1421085 or rs8050136 in iPSC-derived human neurons, suggesting that IRX3/IRX5 function may not be affected by these two SNPs in the CNS.

In conclusion, the data presented here and elsewhere (18, 19, 21) point to risk alleles at SNP rs8050136 and/or rs1421085 that cause decreased RPGRIP1L, FTO, and AKTIP expression by enhancing the binding affinity of P200 (FTO repressor) and diminishing the binding affinity of P110 (transcriptional activator of FTO and RPGRIP1L). The functional consequences of these risk alleles with regard to CUX1 isoform binding mimic the fasting state in which hypothalamic cathepsin L activity is diminished, resulting in decreased cleavage of P200 to P110, thereby causing relatively increased P200 and decreased P110 protein levels (Figure 6). RPGRIP1L/FTO hypomorphism has additive effects on energy intake, resulting in increased adiposity. RPGRIP1L hypomorphism increases food intake via diminished leptin sensitivity. The mechanism(s) underlying the contribution of FTO hypomorphism to increased energy intake in Rpgrip1 $1 \mathrm{l}^{+-} \mathrm{Fto}^{+/-}$mice remains unclear; however, this process may be mediated by demethylase activity controlling translation (48). Last, our data suggest consequences for energy intake in hypothalamic RPGRIP1L/FTO hypomorphism. In view of previous reports in the literature, it is quite likely that multiple mechanisms are at play, in agreement with the statistical signal strength for this interval across ethnic groups. Therefore, the effects of FTO intronic SNPs (not necessarily affecting CUX1 binding) on energy homeostasis may likely extend to other genes (e.g., IRX3, $I R X 5)$ and other tissues, with aggregate mediation of the strong obesity association signal at the FTO locus in humans.

\section{Methods}

Mouse models. Mice carrying the Flp recombinase allele under the $\beta$-actin promoter [C57BL/6Ntac-TG(CAG-Flpe); purchased from Taconic] were bred with mice heterozygous for the Rpgrip1l floxed $L a c Z$ allele (21) to excise the $L a c Z$ cassette. Compound heterozygous Lepr- $b^{\text {Cre/+,Gt(ROSA)26Sortm2Sho/+ }}$ mice have been described elsewhere (21). The Pomc promoter driving expression of Cre recombinase was a gift from Joel Elmquist (University of Texas Southwestern Medical Center, Dallas, Texas, USA). Pomc-GFP [Tg(Pomc1-hrGFP)1Lowl] mice were 
a gift from Bradford Lowell (Beth Israel Deaconess Medical Center, Boston, Massachusetts, USA). The P200- and P110-overexpressing mice, provided by Alain Nepveu (McGill University, Québec, Ontario, Canada), were backcrossed to the C57BL/6J background for 11 generations. Mice segregating for the FTO-floxed allele were provided by Pumin Zhang (Baylor College of Medicine). Mice segregating for the AC3-null allele were provided by Daniel Storm (University of Washington). Finally, we acquired mice hemizygous for an inducible Nestin Cre allele [C57BL/6-Tg(Nes-cre/ERT2)Keisc/J stock number 016261] from The Jackson Laboratory. All mutations were maintained on a C57BL/6J background. Room temperature was constant at $23^{\circ} \mathrm{C}$, a 12 -hourlight/12-hour-dark cycle (lights off at $7 \mathrm{pm}$ ) was used, and mice had ad libitum access to food and water. Animals were maintained according to Columbia University animal welfare guidelines.

Diet and dietary treatment. Male Hprt-P2OO mice $(n=7), H p r t-P 110$ mice $(n=9)$, and control $(+/+)$ littermates $(n=8$ and $n=10$, respectively) were fed a LFD ( $9 \%$ Kcal from fat; Picolab 5058; Purina Mills)

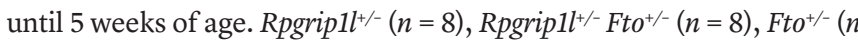
$=9)$, and $+/+$ control mice $(n=12)$ were fed LFD until 14 weeks of age. An additional $6 \mathrm{Fto}^{+/-}, 4$ Rpgripll ${ }^{+/-} \mathrm{Fto}^{+/}$, and 6+/+ male mice were fed LFD chow ad libitum until 14 weeks of age. Eight male Rpgrip1 ${ }^{\text {hyp(Pomc) }}$ mice and ten Rpgrip $1 l^{l / f l}$ littermates were fed LFD for 16 weeks. Eight Rpgrip $1 l^{\text {hyp(Lepr-b) }}$ mice and eight Rpgrip1 $1 l^{\text {llfl }}$ littermates were fed LFD

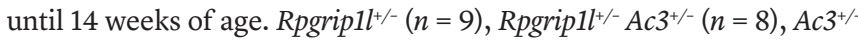
$(n=9)$, and $+/+$ control mice $(n=9)$ were fed LFD until 14 weeks of age and switched to high-fat diet (catalog D12492i, Research Diets; 60\% of kcals from fat) for 4 weeks. An additional $5 \mathrm{Ac}^{+/-}, 5 \mathrm{Rpgrip}^{+/-} \mathrm{Ac3}^{+/-}$, and $6+/+$ male mice were fed LFD ad libitum until 14 weeks of age. Finally, 9 Rpgrip $1 l^{f / f l}$ mice and 11 Rpgrip1 $1 l^{l / f l}$ mice hemizygous for the Nestin-CreER ${ }^{\mathrm{T} 2}$ allele were administered tamoxifen $(225 \mu \mathrm{g}$ per $\mathrm{g}$ of body mass) i.p. 3 times over a 5-day period at the age of 12 weeks.

Body mass, composition measurements, and calorimetry. Food intake and energy expenditure were assessed using the TSE LabMaster system. Body composition was measured with a Minispec TD-NMR Analyzer (Bruker Optics), which was calibrated using mouse carcasses. A detailed description can be found elsewhere (21).

Promoter probing. The putative enhancer, including rs1421085, was PCR amplified using primers (5'-GGGGCCCGGGTTAGATACAGTGGTCTGAGGTCACCTTTG-3' [SmaI] and 5'-CATACCCGGGCCCCAAAATGACTCTGTGCTCATAAGC-3' [SmaI]) and cloned into the $K p n I \mathrm{~N}$-filled and NheI $\mathrm{N}$-filled sites in pGL3-based construct carrying the minimal promoters FTO1p and RPGRIP1Lp, respectively (19). The putative enhancer was also cloned in the $N$-filled KpnI site of pGL3. The luciferase assay was conducted as described elsewhere (19).

Western blotting. P200 and P110 protein levels were assessed in hypothalamic nuclear extracts using anti-CDP (1:250; catalog sc-6327X; Santa Cruz Biotechnologies) via Western blotting, as described elsewhere (19).

FACS. Micropunched whole hypothalami from 4-week-old mice were minced with razor blades and pooled in Hibernate A (catalog HA-Lf; BrainBits). Cells were centrifuged for 1 minute at $400 \mathrm{~g}$, resuspended in papain solution (catalog LK003150, Worthington), and incubated for 10 minutes at $37^{\circ} \mathrm{C}$. Cells were then placed in Hibernate $\mathrm{A}$ and further dissociated by trituration. Nondissociated cells were removed by running them through a $40-\mathrm{mm}$ filter. Hypothalamic cell suspensions were sorted for NeuN (catalog FCMAB317PE; EMD MILLIPORE), tdTomato, or GFP. iPSC-derived neuronal cultures were dissociated with trypsin (TrypLE, catalog 12605036; Thermo Fisher Scientific) and sorted for CD56, a nerve cell adhesion molecule (catalog 560360; BD), and controlled for CD133, a stem cell/progenitor surface marker (catalog 130-080-801; Miltenyi Biotec). The antiNestin antibody was purchased from Sigma-Aldrich (catalog N5413$100 \mu$; Sigma-Aldrich).

EMSA. EMSA of P200 and P110 at rs1421085 was performed as described elsewhere (19) using the following double-stranded oligonucleotides: 5'-CCTAAGGCATGATATTGATTAAGTGT-3' and 5'-CCTAAGGCATGACATTGATTAAGTGT-3'.

Leptin sensitivity assay. A leptin sensitivity test was performed on Rpgripll ${ }^{+-} \mathrm{Fto}^{+/-}(n=8)$ and $+/+$ control mice $(n=8), \mathrm{Fto}^{+/-}(n=8)$ and $+/+$ control mice $(n=8)$, Rpgrip1 $l^{\text {hyp(Lepr-b) }}(n=8)$ and Rpgrip $1 l^{l / f l}$ litter-

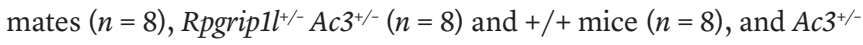
$(n=8)$ and $+/+$ mice $(n=8)$. All mice were 5 - to 6 -week-old males fed LFD ad libitum. Mice were weighed, and their body composition was determined before they were individually caged in a LabMasterCaloSys-Calorimetry System (TSE Systems) and allowed to acclimate in the chambers for the first 24 hours. Four mice of each group were administered saline and four were administered leptin $(4 \mu \mathrm{g} / \mathrm{g})$ at the start of the dark cycle. Food intake and energy expenditure were determined every 26 minutes by the calorimeter. The experiment was repeated with an additional 4 mice in each group, and data were pooled for $n=8$ for each group.

Reprogramming and iPSC culture. Fourteen iPSC lines (4 C/C-A/A, 4 C/T-C/A, 6 T/T-C/C at rs1421085-rs8050136) were established from primary fibroblasts using retroviral reprogramming (49), Sendai virus reprogramming (50), or mRNA reprogramming. For mRNA reprogramming, the Stemgent mRNA Reprogramming Kit (catalog 00-0071) plus miRNA mixture was used. 15,000 to 30,000 fibroblasts were plated on gelatin-coated tissue culture 12-well dishes. 24 to 48 hours after seeding fibroblasts, miRNA cocktail was added to the fibroblasts at day $\mathrm{O}$ of reprogramming. The mRNA cocktail consisting of Oct4, Sox2, Klf4, C-Myc, Lin28, and nGFP was added at days 1 to 3. On day 4, both miRNA and mRNA cocktails were added. On days 5 to 11 , the reprogramming fibroblasts were treated with the mRNA cocktail only. iPSC colonies in the fibroblast culture were handpicked and transferred to a 24-well dish coated with mouse embryonic fibroblasts (MEFs); colonies were transferred to individual wells. Once colonies reached confluence, they were serially expanded. Sendai virus reprogramming was performed using the CytoTune- iPS Sendai Reprogramming Kit (Invitrogen) as previously described (49). Human iPSCs were plated onto MEFs (CF-1 MEF IRR; Globalstem) and cultured in human ES media (KO-DMEM, 10\% KO-SR, 1\% NEAA, 1\% Glutamax, $0.1 \% \mathrm{~b}-\mathrm{ME}, 1 \% \mathrm{P} / \mathrm{S}$, and $10 \mathrm{ng} / \mathrm{ml} \mathrm{bFGF}$ ). Media were supplemented with Y-27632 upon passaging.

Teratoma analysis. About 250,000 undifferentiated iPSCs were dissociated using trypsin (TrypLE, catalog 12605036; Thermo Fisher Scientific) and were spun down at $0.8 \mathrm{RCF}$ for 4 minutes and then resuspended in $50 \mu \mathrm{l}$ human ES media with Y-27632. Cells were mixed 1:1 (vol/vol) in Matrigel (catalog 356231; Corning) and were injected subcutaneously into the dorsal flanks of NSG immunocompromised mice. Cells were allowed to grow for approximately 12 weeks; mice were monitored twice weekly for the appearance of growths and signs of distress. Mice were sacrificed, and teratomas were removed, fixed overnight in $4 \%$ PFA, paraffin embedded, sectioned, and stained with hematoxylin and eosin. 
Neuronal differentiation. Neuronal reprogramming was performed in technical duplicates or triplicates for each established iPSC line using a modified dual SMAD protocol (51). Undifferentiated iPSCs grown on MEFs were dissociated and replated onto fresh MEFs at a density of 200,000 cells per well of a 6-well plate in human ES media with Y-27632 day 0. On day 1, media were changed to EB media (HES media without bFGF) supplemented with $10 \mu \mathrm{M}$ SB431542 and 250 nM LDN193189 (LSB; catalog 5004; Axon). Cells were treated with EB media plus LSB on days 2 and 3; 70\% media changes were performed each day. On day $4,75 \%$ EB media plus 25\% N2 (DMEM/F12 with 1× N2 supplement, 1\% ml GlutaMAX, 1\% $\mathrm{ml}$ penicillin/streptomycin, $0.2 \mathrm{mM}$ ascorbic acid, and $1.6 \%$ glucose) with LSB was used. On day 5, 50\% EB media with 50\% N2 with LSB was used. On day 6, 25\% EB media plus 75\% N2 with LSB was used. $\mathrm{N} 2$ supplemented with $\mathrm{EB}$ media was used on days 7 to 10 . On day 10 , cells were dissociated and plated onto poly-L-ornithine- and laminin-coated plates in N2 plus LSB media with Y-27632. 200,000 cells per well for a 6 -well plate or 80,000 cells per well for a 12-well plate were seeded. On day 11, the media were changed to N2 plus B27 without retinoic acid and $20 \mathrm{ng} / \mu \mathrm{l}$ recombinant BDNF (catalog 248-BD; R\&D Systems). Neuron progenitors were allowed to mature until day 33 or day 34, at which point they were dissociated using Accutase (catalog A1110501; Thermo Fisher Scientific) and FACS sorted for the neural marker NCAM (CD56; catalog 318309; Biolegend). CD56-positive iPSC-derived neurons were pelleted and RNA was isolated using the Norgen Biotek Total RNA Purification Micro Kit (catalog 35300) with DNAse treatment.

Immunocytochemistry. To detect expression of canonical pluripotency markers, iPSC lines of at least passage 10 were fixed in $4 \%$ PFA for 10 minutes, washed with PBS twice, blocked for 1 hour at room temperature using 10\% donkey serum in 0.1\% PBST, washed with PBS 3 times, incubated in primary antibody overnight at $4^{\circ} \mathrm{C}$, washed with PBS 3 times, and incubated with secondary antibody at room temperature for 2 hours. Primary antibodies used to detect pluripotency are as follows: Oct4 (1:500; catalog 09-0023; Stemgent), Tra1-60 (1:500; catalog MAB4360; Millipore), Tra1-81 (1:500; catalog MAB4381; Millipore), and Nanog (1:300; catalog D73G4; Cell Signaling Technologies). All Alexa Fluor secondary antibodies were diluted 1:1,000. Hoechst 33342 (catalog B2261; Sigma-Aldrich) was used to mark nuclei. iPSC-derived neurons were stained with the following neural makers: MAP2 (catalog ab5392; Abcam), TUJ1 (catalog T2200; Sigma-Aldrich), NF (catalog 70748; Millipore), and Nestin (catalog MAB353; Millipore). The staining procedure was the same as for iPSCs.

Quantitative PCR. For expression analysis, Hprt-P200, Hprt-P110,

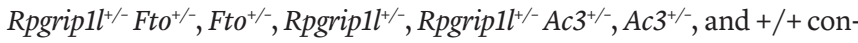
trol mice (each group $n=5$ ) were sacrificed. Whole brains were placed on a dissection block, and hypothalami were dissected from the rostral border of the optic chiasm to the rostral border of the mammillary body. Fto, Rpgrip1l, Npy, Pomc, Pmch, AgRP, Cdh9, Rbl2, Aktip, Irx3, Irx5, and $A d c y 3$ mRNA levels were measured by quantitative PCR as described elsewhere (18). Transcript levels of FTO (5'-GAGCTTGAAGACACTTGGCTCCC-3', 5'-GCAGCCATGCTTGTGCAGTGTGA-3'), RPGRIP1L (5'-CCATGGATCAAGCAATTCGACTTTATCGAG-3', 5'-GGGACTGCAGGTGGTTGCAAC-3'), AKTIP (5'-GCAGCACAGTCAACAAATGGCACG-3', 5'-GCCATCTTGGTAAAGTCCATGCCG-3'), IRX3 (5'-CCCTGTCCAACGTGCTCTCGTC-3',
5'-GCTGTCCTTCAGCTCATACTGCG-3'), RBL2 (5'-GGGACAGCTACCGCAGCATG-3', 5'-GGGTAGATTTGCCATGTCTTCCCAC-3'), IRX5 (5'-CAACTCGCACCTCCAGTACGG-3', 5'-GTCCCTTGTGGCGTTCTTCCG-3'), and CHD9 (5'-GGCTCAGACTCAGTTGCAAAGTCAGG-3', 5'-GGCTCAGACTCAGTTGCAAAGTCAGG-3') in human iPSC-derived neurons were also assessed by quantitative PCR. mRNA levels were normalized as described elsewhere (18).

Genotyping. The P200 and P110 human transgenes were detected using the following primers: 5'-GAAGGAGGCGCTGAAACGAGCG-3', 5'-CTCAATGAACAGTTCTCTGCGGATCC-3'. The Rpgrip1l floxed (Rpgrip $\left.1 l^{f}\right)$ and deleted alleles were detected using the following primers: 5'-GTCTTGGACAGATCTTGGTCCAGTCTC-3'， 5'-GTGGGTTGTACAGTTTCTGCTTCATCC-3' (Rpgrip1 $\left.l^{\prime \prime}\right)$, 5'-GCCTTCCATATAACTGGCCAGTTGTTTC-3', 5'-GGATGTGGCAGAAGGATTGTTTCTGAGA-3' (excised).

The following primers were used to assess the presence of the Fto-null and wild-type alleles: 5'-TTACTTCATGCCAGGGGTTCTGATTCG-3', 5'-CGACAATCGAGATGGTGATGTCAGG-3', 5'-CAGCACAGTGGTCTGAGGACAAGCA-3'. Mice carrying the Lepr-b Cre recombinase knockin or wild-type alleles were genotyped using the following primers: 5'-TCGTGTTGAAATTTCTTCTTTCCAGA-3', 5'-TCCAAGAAGCCTCAAGGTTCCA-3' (wild type), 5'-ACGCACACCGGCCTTATTCC-3', 5'-TCCAAGAAGCCTCAAGGTTCCA-3' (knockin). The Pomc-Cre recombinase transgene was detected using the following primers: 5'-TTACATTGGTCCAGCCACC, 5'-ACCAGCCAGCTATCAACTCG-3'. Finally, primers 5'-ATCCGGCTACCTGCCCATTCGACC-3' and 5'-TTGAGCCTGGCGAACAGTTCGGCTGG-3' were used to detect the AC3 knockout allele, whereas primers 5'-CCAGCAGCCAACATGCCGAGGAA-3' and 5'-ACGATGGGGCTGAGGCTGAGGGGC-3' were used to detect the AC3 wild-type allele.

Statistics. Statistical significance was determined by a 2-tailed paired Student's $t$ test. $P$ values lower than 0.05 were considered significant. ANOVA analysis was carried out using JMP. We created a baseline regression equation for control mice at 5 weeks of age fed LFD ad libitum, relating food intake (FI; g/24 h) or energy expenditure $(\mathrm{EE} ; \mathrm{kcal} / 24 \mathrm{~h})$ to fat mass $(\mathrm{FM})$ and lean mass $(\mathrm{LM})(\mathrm{g})(\mathrm{FI}=$ $-0.52 \times \mathrm{FM}+0.44 \times \mathrm{LM}-3.8, \mathrm{R}^{2}=0.77, P<0.01 ; \mathrm{EE}=1.18 \times \mathrm{FM}+0.49$ $\left.\times \mathrm{LM}-1.4, \mathrm{R}^{2}=0.82, P<0.01\right)$. This equation was used to generate predicted values for Hprt-P2OO and Hprt-P110 mice. We also used the following baseline regression equations to relate food intake and energy expenditure of Rpgrip 1 hyp(Nestin) $^{\text {mice }}(\mathrm{FI}=0.1 \times \mathrm{FM}+0.1 \times \mathrm{LM}$ $-0.1, \mathrm{R}^{2}=0.9, P<0.01 ; \mathrm{EE}=-0.2 \times \mathrm{FM}+0.56 \times \mathrm{LM}-0.4, \mathrm{R}^{2}=0.87$, $P<0.01)$ and Rpgrip1 hyp(Pomc) mice $\left(\mathrm{FI}=0.76 \times \mathrm{FM}-0.07 \times \mathrm{LM}-1, \mathrm{R}^{2}=\right.$ $\left.0.96, P<0.01 ; \mathrm{EE}=0.58 \times \mathrm{FM}-0.16 \times \mathrm{LM}+011.8, \mathrm{R}^{2}=0.87, P<0.01\right)$ to fat mass and lean mass.

Study approval. Human subject research (written informed consent provided) was reviewed and approved by the Columbia Stem Cell Committee and the Columbia IRB. All animal studies were approved by the Columbia IACUC.

\section{Author contributions}

GS designed and conducted experiments, acquired data, analyzed data, and partly wrote the manuscript. LCB acquired data and helped write the manuscript. RR acquired data. RG critiqued and helped write the manuscript. DBP and AJL acquired data. AAS and CAL acquired data and helped write the manuscript. DRS and PZ provided reagents. DE provided reagents, supervised LCB, and 
designed experiments. RLL designed experiments, analyzed data, and partly wrote the manuscript.

\section{Acknowledgments}

We would like to thank Alain Nepveu (McGill University) for useful discussion and for donating the Hprt-P2OO and Hprt-P11O mice; Daniel R. Storm (University of Washington) for providing mice segregating for the Ac3 knock out allele; and Pumin Zhang (Baylor College of Medicine) for donating mice segregating for the Fto- floxed allele. This work was funded by NIH grants R01 DK52431, P30DK026687, and DC004156; The Moses Lasky Foundation; and The Russell Berrie Foundation.

Address correspondence to: Rudolph L. Leibel or George Stratigopoulos, 1150 St. Nicholas Ave., RM 620, New York, New York 10032, USA. Phone: 212.851.5257; E-mail: RL232@columbia. edu (R.L. Leibel), Phone: 212.851.5309; GS2172@columbia.edu (G. Stratigopoulos).
1. Frayling TM, et al. A common variant in the FTO gene is associated with body mass index and predisposes to childhood and adult obesity. Science. 2007;316(5826):889-894.

2. Berulava T, Horsthemke B. The obesity-associated SNPs in intron 1 of the FTO gene affect primary transcript levels. Eur J Hum Genet. 2015;18(9):1054-1056.

3. Jowett JB, et al. Genetic variation at the FTO locus influences RBL2 gene expression. Diabetes. 2010;59(3):726-732.

4. Smemo $\mathrm{S}$, et al. Obesity-associated variants within FTO form long-range functional connections with IRX3. Nature. 2014;507(7492):371-375.

5. Claussnitzer M, et al. FTO obesity variant circuitry and adipocyte browning in humans. $N$ Engl JMed. 2015;373(10):895-907.

6. Gerken T, et al. The obesity-associated FTO gene encodes a 2-oxoglutarate-dependent nucleic acid demethylase. Science. 2007;318(5855):1469-1472.

7. Hess $\mathrm{M}$, et al. The fat mass and obesity associated gene (Fto) regulates activity of the dopaminergic midbrain circuitry. Nat Neurosci. 2013;16(8):1042-1048.

8. Karra E, et al. A link between FTO, ghrelin, and impaired brain food-cue responsivity. J Clin Invest. 2013;123(8):3539-3551.

9. Zhang M, et al. The demethylase activity of FTO (fat mass and obesity associated protein) is required for preadipocyte differentiation. PLoS One. 2015;10(7):e0133788.

10. Meyre D, et al. Prevalence of loss-of-function FTO mutations in lean and obese individuals. Diabetes. 2010;59(1):311-318.

11. Deliard S, et al. The missense variation landscape of FTO, MC4R, and TMEM18 in obese children of African Ancestry. Obesity (Silver Spring). 2013;21(1):159-163.

12. Zheng $\mathrm{Z}$, et al. Screening for coding variants in FTO and SH2B1 genes in Chinese patients with obesity. PLoS One. 2013;8(6):e67039.

13. Qi Q, et al. Dietary intake, FTO genetic variants, and adiposity: a combined analysis of over 16,000 children and adolescents. Diabetes. 2015;64(7):2467-2476

14. Haupt A, et al. Variation in the FTO gene influences food intake but not energy expenditure. Exp Clin Endocrinol Diabetes. 2009;117(4):194-197.

15. Speakman JR, Rance KA, Johnstone AM. Polymorphisms of the FTO gene are associated with variation in energy intake, but not energy expenditure. Obesity (Silver Spring). 2008;16(8):1961-1965.

16. Jensen VL, et al. Formation of the transition zone by Mks5/Rpgrip1L establishes a ciliary zone of exclusion (CIZE) that compartmentalises ciliary signalling proteins and controls PIP2 ciliary abundance. $E M B O$ J. 2015;34(20):2537-2556.

17. Oh EC, Vasanth S, Katsanis N. Metabolic regulation and energy homeostasis through the primary Cilium. Cell Metab. 2015;21(1):21-31.

18. Stratigopoulos G, et al. Regulation of Fto/Ftm gene expression in mice and humans. Am JPhysiol Regul Integr Comp Physiol. 2008;295(4):R1360-R1363.

19. Stratigopoulos G, LeDuc CA, Cremona ML, Chung WK, Leibel RL. Cut-like homeobox 1 (CUX1) regulates expression of the fat mass and obesity-associated and retinitis pigmentosa GTPase regulator-interacting protein-1-like (RPGRIP1L) genes and coordinates leptin receptor signaling. J Biol Chem. 2011;286(3):2155-2170.

20. Moon NS, et al. S phase-specific proteolytic cleavage is required to activate stable DNA binding by the CDP/Cut homeodomain protein. Mol Cell Biol. 2001;21(18):6332-6345.

21. Stratigopoulos G, et al. Hypomorphism for RPGRIP1L, a ciliary gene vicinal to the FTO locus, causes increased adiposity in mice. Cell Metab. 2014;19(5):767-779.

22. Fischer J, et al. Inactivation of the Fto gene protects from obesity. Nature. 2009;458(7240):894-898.

23. Peters U, et al. A systematic mapping approach of 16q12.2/FTO and BMI in more than 20,000 African Americans narrows in on the underlying functional variation: results from the Population Architecture using Genomics and Epidemiology (PAGE) study. PLoS Genet. 2013;9(1):e1003171.

24. Gong J, et al. Fine mapping and identification of BMI loci in African Americans. Am J Hum Genet. 2013;93(4):661-671.

25. Hassanein MT, et al. Fine mapping of the association with obesity at the FTO locus in African-derived populations. Hum Mol Genet. 2010;19(14):2907-2916.

26. Aufiero B, Neufeld EJ, Orkin SH. Sequencespecific DNA binding of individual cut repeats of the human CCAAT displacement/cut homeodomain protein. Proc Natl Acad Sci U S A. 1994;91(16):7757-7761.

27. Ramdzan ZM, et al. RAS transformation requires CUX1-dependent repair of oxidative DNA damage. PLoS Biol. 2014;12(3):e1001807.

28. Cadieux C, et al. Mouse mammary tumor virus p75 and p110 CUX1 transgenic mice develop mammary tumors of various histologic types. Cancer Res. 2009;69(18):7188-7197.

29. Gao X, et al. The fat mass and obesity associated gene FTO functions in the brain to regulate postnatal growth in mice. PLoS One. 2010;5(11):e14005.

30. Boisse S, et al. Loss-of-function mutation in the dioxygenase-encoding FTO gene causes severe growth retardation and multiple malformations. Am JHum Genet. 2009;85(1):106-111.

31. Daoud $\mathrm{H}$, et al. Identification of a pathogenic FTO mutation by next-generation sequencing in a newborn with growth retardation and developmental delay. JMed Genet. 2015;53(3):200-207.

32. Wang $Z$, et al. Adult type 3 adenylyl cyclase-deficient mice are obese. PLoS One. 2009;4(9):e6979.

33. Minokoshi Y, et al. Leptin stimulates fatty-acid oxidation by activating AMP-activated protein kinase. Nature. 2002;415(6869):339-343.

34. Dhar M, et al. Leptin induces hippocampal synaptogenesis via CREB-regulated microRNA-132 suppression of p250GAP. Mol Endocrinol. 2014;28(7):1073-1087.

35. Remy I, Michnick SW. Regulation of apoptosis by the $\mathrm{Ft} 1$ protein, a new modulator of protein kinase B/Akt. Mol Cell Biol. 2004;24(4):1493-1504.

36. Lin YW, Yan MD, Shih YL, Hsieh CB. The basal body gene, RPGRIP1L, is a candidate tumour suppressor gene in human hepatocellular carcinoma. Eur JCancer. 2009;45(11):2041-2049.

37. Kokoeva MV, Yin H, Flier JS. Neurogenesis in the hypothalamus of adult mice: potential role in energy balance. Science. 2005;310(5748):679-683.

38. Goldenberg N, Barkan A. Factors regulating growth hormone secretion in humans. Endocrinol Metab Clin North Am. 2007;36(1):37-55.

39. Iwanaga T, Miki T, Takahashi-Iwanaga $H$. Restricted expression of somatostatin receptor 3 to primary cilia in the pancreatic islets and adenohypophysis of mice. Biomed Res. 2011;32(1):73-81.

40. Gulati P, et al. Role for the obesity-related FTO gene in the cellular sensing of amino acids. Proc Natl Acad Sci U S A. 2013;110(7):2557-2562.

41. Osborn DP, et al. Loss of FTO antagonises Wnt signaling and leads to developmental defects associated with ciliopathies. PLoS One. 2014;9(2):e87662.

42. Tung YC, et al. Hypothalamic-specific manipulation of Fto, the ortholog of the human obesity gene FTO, affects food intake in rats. PLoS One. 2010;5(1):e8771.

43. Wang P, et al. Involvement of leptin receptor long isoform (LepRb)-STAT3 signaling pathway in brain fat mass- and obesity-associated (FTO) downregulation during energy restriction. $\mathrm{Mol}$ Med. 2011;17(5-6):523-532.

44. Church C, et al. Overexpression of Fto leads to increased food intake and results in obesity. Nat Genet. 2010;42(12):1086-1092.

45. McMurray F, et al. Adult onset global loss of the 


\section{RESEARCH ARTICLE}

fto gene alters body composition and metabolism in the mouse. PLoS Genet. 2013;9(1):e1003166.

46. Arts HH, et al. Mutations in the gene encoding the basal body protein RPGRIP1L, a nephrocystin- 4 interactor, cause Joubert syndrome. Nat Genet. 2007;39(7):882-888.

47. Thomas S, et al. Identification of a novel ARL13B variant in a Joubert syndrome-affected patient with retinal impairment and obesity. Eur J Hum
Genet. 2015;23(5):621-627.

48. Zhou J, Wan J, Gao X, Zhang X, Jaffrey SR, Qian SB. Dynamic m(6)A mRNA methylation directs translational control of heat shock response. Nature. 2015;526(7574):591-594.

49. Fusaki N, Ban H, Nishiyama A, Saeki K, Hasegawa M. Efficient induction of transgene-free human pluripotent stem cells using a vector based on Sendai virus, an RNA virus that does
The Journal of Clinical Investigation

not integrate into the host genome. Proc Jpn Acad Ser B Phys Biol Sci. 2009;85(8):348-362.

50. Takahashi K, et al. Induction of pluripotent stem cells from adult human fibroblasts by defined factors. Cell. 2007;126(4):663-676.

51. Chambers SM, et al. Highly efficient neural conversion of human ES and iPS cells by dual inhibition of SMAD signaling. Nat Biotechnol. 2009;27(3):275-280. 